Available online at:

https://acta-acustica.edpsciences.org

\title{
Acoustics of the banjo: theoretical and numerical modelling
}

\author{
Jim Woodhouse ${ }^{1, *}$, David Politzer ${ }^{2}$, and Hossein Mansour ${ }^{3}$ \\ ${ }^{1}$ Cambridge University Engineering Department, Trumpington Street, Cambridge CB2 1PZ, UK \\ ${ }^{2}$ Caltech 452-48, Pasadena, CA 91125, USA \\ ${ }^{3}$ Dassault Systèmes - SIMULIA, 5005 Wateridge Vista Dr., San Diego, CA 92121, USA
}

Received 17 July 2020, Accepted 17 February 2021

\begin{abstract}
A previous paper [J. Woodhouse et al. Acta Acustica 5, 15 (2021). https://doi.org/10.1051/aacus/ 2021009] showed acoustical measurements of an American 5-string banjo alongside similar measurements on a guitar, revealing a strong contrast in bridge admittance. Theoretical and numerical modelling is now presented to probe the physics behind this contrast. Without the bridge and strings, the banjo membrane has a rising trend of admittance associated with its modal density, and it has a distinctive pattern of sound radiation because an ideal membrane has no critical frequency. When the bridge and strings are added to the banjo, three formants shape the amplitude envelope of the admittance. One is associated with local effects of mass and stiffness near the bridge, and is sensitive to bridge mass and the break angle of the strings over the bridge. The other two formants are associated with dynamical behaviour of the bridge, analogous to the "bridge hill" in the violin.
\end{abstract}

\section{Introduction}

In a preceding paper [1], measurements of vibrational response were presented for an American 5-string banjo (a Deering Eagle II [2]), and contrasted with corresponding results for a steel-string guitar (by Martin Woodhouse [3]). The measured mechanical admittance functions at the bridges of the two instruments were then used as the basis of a synthesis algorithm, to find out whether the differences captured by those measurements were sufficient to allow recognisable and convincing versions of "banjo sound" versus "guitar sound" to be produced. The answer was in the affirmative (supporting sound demonstrations were provided on an accompanying website [4]). The synthesis models were then used to explore the perceptual consequences of a wide range of parametric variations in the banjo. A formant-like feature, peaking around 500-800 Hz on the banjo tested, was found to play an important role in shaping the sound of the banjo. At higher frequencies two additional formant-like features were seen, reminiscent of the "bridge hill" of the violin [5-7].

The agenda for the present paper is to investigate the physics lying behind the contrast in the admittance functions, and to understand the origin of the three banjo formants. A combination of theoretical analysis and numerical modelling will be used. The study divides naturally in two stages. Some key differences between the banjo and guitar admittance are associated directly with the fact that a banjo uses a stretched membrane rather than a wooden

*Corresponding author: jw12@cam.ac.uk box with vibration associated with bending plates. These issues are discussed in Section 2. The extra changes when the banjo bridge and strings are added will be addressed in Section 3.

Among other things, a detailed model based on coupled Finite Elements and Boundary Elements (FE/BE) will be presented. However, it should be emphasised that the objective is understanding, which is not the same thing as the ability to compute a facsimile of something. The $\mathrm{FE} / \mathrm{BE}$ model will be important to explicate certain aspects of the physics of the banjo, but it is not in itself the main objective of this study. Many key ideas can be illustrated neatly and simply using a model based on rectangular membrane geometry. It is suggested that almost any real or synthesised stringed instrument with a soundboard with membrane-like rather than plate-like behaviour can "sound like a banjo", and the rectangular model will be used to lend support to this idea.

Measured admittance functions for the banjo and guitar are shown in Figure 1, for excitation near the bridge notch for the first string. Also included is the admittance measured directly on the head membrane of the banjo, with the strings and bridge removed, at approximately the same position. The three curves in this figure show remarkably different trends and levels: the admittance of the complete banjo lies typically $20-30 \mathrm{~dB}$ above that of the guitar over most of the range, and the admittance of the bare membrane lies a further 20-30 dB higher for frequencies above about $2 \mathrm{kHz}$. Two of the three formants can be seen in the red curve in this plot: a broad feature peaking around 500 $800 \mathrm{~Hz}$, and a smaller peak around $3.5 \mathrm{kHz}$. Some parameter 


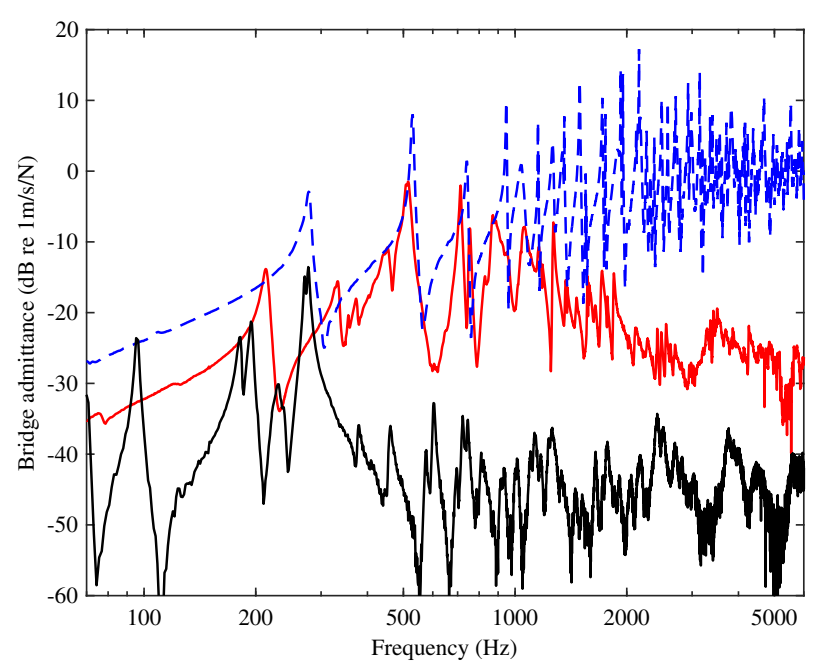

Figure 1. Measured bridge admittance of the banjo (red line) and the guitar (black line). Blue dashed line: admittance of the banjo head without bridge and strings.

Table 1. Parameter values for the Deering banjo. Head tension is estimated from computer modelling: see Section 2.3.

\begin{tabular}{lll}
\hline Head diameter & $d$ & $268 \mathrm{~mm}$ \\
Head thickness & $h$ & $0.18 \mathrm{~mm}$ \\
Mass per unit area & $\sigma$ & $0.30 \mathrm{~kg} / \mathrm{m}^{2}$ \\
Tension & $T$ & $5.33 \mathrm{kN} / \mathrm{m}$ \\
Bridge mass & $m_{b}$ & $2.2 \mathrm{~g}$ \\
Bridge footprint & & $78 \times 5 \mathrm{~mm}$ \\
\hline
\end{tabular}

values for the tested banjo are listed in Table 1. Figure 1 of the previous paper [1] included a cross-sectional diagram of the banjo body, showing the configuration of the pot and resonator back.

\section{What makes a membrane different?}

The first task is to explain the differences between the blue and black curves in Figure 1. The simplest difference lies in the mass: the total mass of the bare banjo head is about $18 \mathrm{~g}$, compared to the typical mass of a guitar top plate (with braces and bridge) of the order of $200 \mathrm{~g}$. The low mass of the banjo head has direct consequences for the admittance.

Apart from mass, there are two main ways in which the vibrational characteristics of a membrane differ from those of a plate: the modal density, and the coupling to sound waves in the surrounding air (see e.g. [8, 9]). Both will turn out to be crucial to understanding the banjo, and they will be considered in turn. Both effects can be conveniently illustrated by making use of a very simple example model based on rectangular geometry, for which the mode shapes, natural frequencies and sound radiation damping are available in elementary closed form. Of course, the mode shapes of an ideal circular membrane are also known in closed form, in terms of Bessel functions [10], and it might seem more natural to use that more realistic system to model the banjo. However, the corresponding expressions for a circular plate are more complicated, and thus less illuminating, and more importantly there are no analytic expressions for sound radiation for circular geometry.

In fact, it will be suggested that a "square banjo" like this would not sound very different from a normal circular one: indeed, non-circular banjo-like instruments, including rectangular ones, really exist in various corners of the world. In addition, audio demonstrations based on the model to be developed here were presented and discussed in the earlier paper [1] and the website [4]: the examples Sound B.1 and Sound B.2 on that website give a direct comparison between synthesis based on measured admittance and on the square banjo model. Later in the present paper the frequency response behaviour of the model will also be compared with that of the detailed FE/BE numerical model.

\subsection{The role of modal density}

Idealised theoretical models for the vibration of a stretched membrane and a bending plate are very familiar: see for example Rayleigh [10]. The most obvious difference is that membrane vibration is governed by a second-order differential equation, whereas plate vibration involves fourth-order derivatives in the spatial coordinates. This has an immediate consequence for the dispersion relation and modal density of the two systems, giving rise to qualitative differences of behaviour that are independent of detailed shapes and boundary conditions.

A rectangular membrane of dimensions $a \times b$ has exactly the same mode shapes as a rectangular plate of the same dimensions with hinged boundary conditions around the edges. The $(n, m)$ th mode shape is

$$
u_{n m}=K \sin \left(\frac{n \pi x}{a}\right) \sin \left(\frac{m \pi y}{b}\right),
$$

where $x, y$ are coordinates in the plane, $n$ and $m$ can take any positive integer values, and the constant

$$
K=\frac{2}{\sqrt{\sigma a b}},
$$

enforces the usual mass-normalisation (see e.g. [11]), where $\sigma$ is the mass per unit area of the membrane or plate. This normalisation factor will not be strictly accurate when the membrane/plate is attached to an instrument body with finite mass: the modes need to be orthogonal to the rigidbody modes of the entire instrument, so the body mass enters the calculation. However, in the limit when the mass ratio of body to membrane/plate is large, the correction tends to zero. The mass of the Deering banjo is approximately $4.5 \mathrm{~kg}$ compared to a head mass around $20 \mathrm{~g}$, so the mass ratio is large enough for this limiting case to apply very accurately.

The corresponding natural frequency of the membrane is

$$
\omega_{n m}^{(\mathrm{m})}=\sqrt{\frac{T}{\sigma}\left[\left(\frac{n \pi}{a}\right)^{2}+\left(\frac{m \pi}{b}\right)^{2}\right]},
$$


while that of the plate is

$$
\omega_{n m}^{(\mathrm{p})}=\sqrt{\frac{E h^{3}}{12 \sigma\left(1-v^{2}\right)}}\left[\left(\frac{n \pi}{a}\right)^{2}+\left(\frac{m \pi}{b}\right)^{2}\right],
$$

where $T$ is the membrane tension, and the plate is assumed to be made of isotropic material with Young's modulus $E$, Poisson's ratio $v$ and thickness $h$.

Because both systems have mode shapes that occupy the same grid of points in $2 \mathrm{D}$ wavenumber space, there is a simple method to compare the modal density for the two systems: see for example $[12,8]$. The result is that the plate, and indeed any plate, has modal density which is (on average) constant with frequency, while any membrane has modal density which increases proportional to frequency. Equivalently, the average modal spacing is constant for a plate (for a typical guitar top, this average spacing is of the order of $60 \mathrm{~Hz}$ ), while for a membrane the spacing reduces as frequency goes up (for the banjo head the spacing is around $50 \mathrm{~Hz}$ at $1 \mathrm{kHz}$, reducing to $10 \mathrm{~Hz}$ at $5 \mathrm{kHz}$ ).

For either system, the input admittance (the velocity for a given force) at a position $\left(x_{b}, y_{b}\right)$ is given by the standard formula

$$
Y\left(x_{b}, y_{b}, \omega\right)=\mathrm{i} \omega \sum_{n, m} \frac{u_{n m}^{2}\left(x_{b}, y_{b}\right)}{\omega_{n m}^{2}+\mathrm{i} \omega \omega_{n m} \eta_{n m}-\omega^{2}},
$$

where the relevant one of equations (3) or (4) is used for the natural frequencies, and $\eta_{n m}$ is the modal loss factor (the inverse of the modal Q-factor). However, a modification is needed to ensure convergence in the case of a membrane. An ideal membrane forced at a single point exhibits a logarithmic singularity of displacement (see for example Sect. 5.2 of Morse and Ingard [13]), which is obviously unrealistic in physical terms. Some consequences of this suppressed singularity will be discussed in Section 3.4. For the moment, a simple way to deal with this is to calculate the admittance corresponding to an average over a finite footprint, rather than to point loading. The mode shape of equation (1) can be averaged in closed form over a rectangular region $x_{b}-x_{f} \leq x \leq x_{b}+x_{f}$, $y_{b}-y_{f} \leq y \leq y_{b}+y_{f}$, to yield

$$
\begin{gathered}
u_{n m}^{(f)}\left(x_{b}, y_{b}\right)=\frac{2 \sqrt{a b}}{n m \pi^{2} x_{f} y_{f} \sqrt{\sigma}} \sin \left(n \pi x_{b} / a\right) \\
\times \sin \left(n \pi x_{f} / a\right) \sin \left(m \pi y_{b} / b\right) \sin \left(m \pi y_{f} / b\right) .
\end{gathered}
$$

This patch-averaged shape reduces to equation (1) in the limit when $x_{f}$ and $y_{f}$ are both small. It can be used in the admittance formula equation (5). The dimensions chosen, somewhat arbitrarily, for the initial plots correspond to a square footprint with half-widths $x_{f}=y_{f}=5 \mathrm{~mm}$.

Figure 2a shows examples, for a deliberately idealised and extreme comparison. To bring out the modal density effect with the minimum of confusion from other phenomena, everything else was held constant. The parameter values are based on the Deering banjo, as given in Table 1. A membrane with the same area, tension and mass per unit area is used, in a shape which is not quite square to avoid
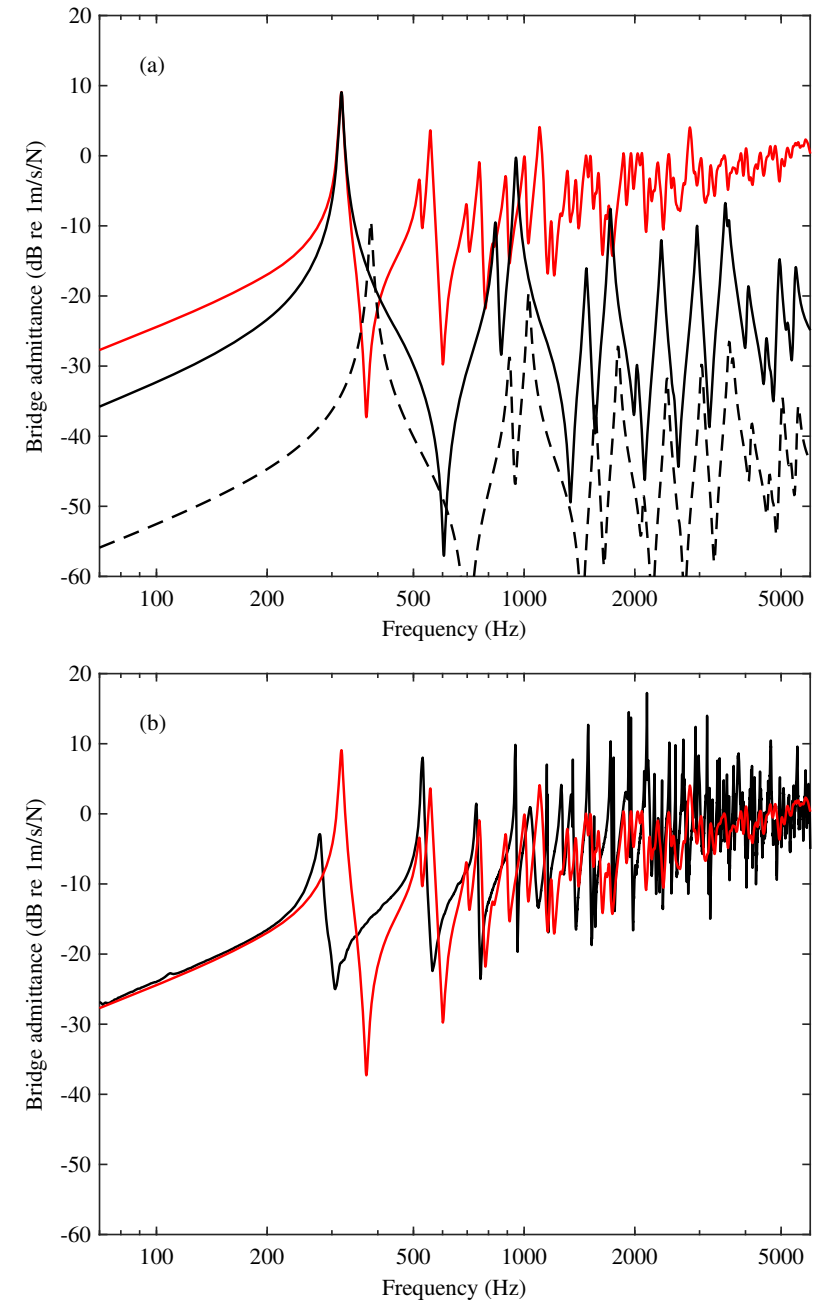

Figure 2. (a) Comparison of synthesised admittances for rectangular membrane (red) and plate (black) with matching fundamental frequency, damping and mass (see text for details). Dashed black line shows the plate with mass increased by a factor 10. (b) Comparison of synthesised admittance (red) as in (a) with the measured admittance of the bare head of the Deering banjo (black).

degeneracies. Specifically, the membrane has dimensions $a=0.249 \mathrm{~m}, b=0.226 \mathrm{~m}$, and the admittance is calculated for the square footprint centred at position $x_{b}=a / 2.37$, $y_{b}=b / 2.84$. The position $y_{b}$ was chosen to reflect, approximately, the bridge position on the banjo, while $x_{b}$ was chosen slightly off-centre to avoid symmetry lines. All modes were assigned the same Q-factor, with value 50 (i.e. $\eta_{n m}=0.02$ ). The plate is identical to the membrane in all parameters other than the frequencies of higher modes: the same geometry, fundamental frequency, mass, damping factor and driving position.

It can be seen in Figure 2a that the membrane and plate have identical behaviour near the fundamental resonance peak, as they are designed to, but everywhere else the curves diverge. The different modal density behaviour is immediately apparent: the membrane has far more modes than the plate at higher frequency, and among other things this 
means that the modal overlap factor grows more rapidly. The two curves also diverge in amplitude: as a direct result of the lower mode count in the plate, the mean level in this decibel plot falls progressively below that of the membrane.

The dashed line in Figure 2a shows the effect of increasing the mass per unit area of the plate by a factor 10, to bring the total mass closer to the kind of value that might be expected for a guitar top plate with braces and bridge. The effect, on the decibel scale, is simply to shift the curve vertically so that the contrast with the membrane case is bigger. The general level of the plate curve at higher frequencies is now comparable to the guitar measurement shown in Figure 1.

Figure $2 \mathrm{~b}$ shows a comparison of the simple rectangular membrane model with the measured response on the banjo head. The general level and trend match well despite the apparent crudeness of the model. The fundamental frequency is significantly lower on the banjo, due largely to an effect of loading by the surrounding air to be discussed in Section 2.2. This peak is significantly taller in the simple model, caused in part by a difference in modal damping associated with sound radiation, also to be discussed in Section 2.2. The same issue of damping also affects the typical peak-to-valley fluctuations at higher frequency: membrane Q's will shortly be shown to grow systematically with frequency, rendering the assumption of a constant $\mathrm{Q}$-factor inappropriate. By contrast, constant $\mathrm{Q}$ gives a fairly good approximation to the behaviour expected of a plate system like a guitar body: the Q-factor used in Figure 2a is comparable with typical damping in a guitar.

The different trends of the various curves in Figures 1 and 2 can be readily explained using statistical approaches to vibration, such as Skudrzyk's mean value method [14] or Statistical Energy Analysis [12]. The simplest illustration is given by Skudrzyk's method, which has been used in previous literature of stringed instrument acoustics $[7,15,16]$. It relies on the fact that in the limit when damping is increased, any driving-point admittance tends towards a smooth curve that is the mean trend of a decibel plot like the ones shown here. When the modal overlap factor is small, the peak amplitudes rise above this mean trend by a factor of the order of the typical Q factor, while the antiresonance levels fall below it by a comparable factor. The high-damping limit has a simple physical interpretation, in terms of an infinitely-extended system with no boundary reflections. So the trends seen in Figure 2a should be predicted by an infinite point-driven plate and an infinite membrane, respectively. In the case of the membrane, a finite footprint must again be used.

The point admittance of an infinite plate matches that of a mechanical resistance, with a positive real value independent of frequency (see e.g. [17]): for the isotropic plate considered here, the result is

$$
Y_{\infty}^{(\mathrm{p})}(\omega)=\frac{1}{8} \sqrt{\frac{12\left(1-v^{2}\right)}{E h^{3} \sigma}} .
$$

For an infinite membrane driven through a circular footprint of radius $a$, the admittance is

$$
Y_{\infty}(a, \omega)=\frac{i c}{2 \pi a T} \frac{\mathrm{H}_{0}^{(2)}(\omega a / c)}{\mathrm{H}_{1}^{(2)}(\omega a / c)},
$$

where $\mathrm{H}_{N}^{(2)}$ is the Hankel function of order $N$ associated with outward-travelling waves with the sign convention in use here, and $c=\sqrt{T / \sigma}$ is the membrane wave speed. Using the asymptotic behaviour of the Hankel functions, there is a very simple limit at high frequency:

$$
Y_{\infty}(a, \omega) \rightarrow \frac{c}{2 \pi a T} \quad \text { as } \quad \omega \rightarrow \infty .
$$

The membrane behaviour tends towards that of a mechanical resistance, but unlike the plate case the value of that resistance depends on the assumed footprint radius $a$.

Figure 3 shows examples of these results, corresponding quantitatively to the cases of Figure 2a, using a footprint radius chosen to give the same area as the square footprint in that figure. The black and blue curves mirror the trends seen in Figure 2 with satisfying accuracy. For the bare membrane, the rising trend at lower frequency is a direct reflection of the increasing modal density of an ideal membrane, and is independent of the footprint size. However, the finite footprint means that the curve flattens out as it approaches the asymptotic constant value (shown as a blue dashed line). The smaller the footprint, the higher the asymptotic value and the higher the frequency of this turn-over.

Skudrzyk's method can also be used to give a first indication of the effect on these trend lines of adding the banjo bridge and strings. The simplest idealisation is to treat the bridge as a concentrated load over the chosen footprint, with mass $m_{b}$. For reasons to be discussed in Section 3.2, there are two other effects that need to be included in the concentrated loading. There is a localised stiffness arising from a combination of string tension and axial stiffness, acting through the geometry of the break angle over the bridge, and a related effect of in-plane stiffness of the membrane. The combined effect can be represented by a spring of stiffness $k_{s}$. Also, additional damping associated with the presence of the bridge and strings can be represented by a mechanical resistance or "dashpot" with impedance $\lambda$. These effects can all be combined using a general result for "point coupling": the modified admittance $Y_{b, \infty}$ satisfies

$$
\frac{1}{Y_{b, \infty}(\omega)}=\frac{1}{Y_{\infty}(\omega)}+\mathrm{i} \omega m_{b}+\frac{k_{s}}{i \omega}+\lambda .
$$

The red curve in Figure 3 shows the predicted effect of adding a mass $m_{b}=1.5 \mathrm{~g}$ to the membrane with the chosen footprint size. Finally, the red dashed curve shows the additional effect of including a stiffness $k_{s}=20 \mathrm{kN} / \mathrm{m}$ and a dashpot with $\lambda=1 \mathrm{Ns} / \mathrm{m}$. The contrast between the blue curve and the red dashed curve mirrors the difference seen in Figure 1 between the bare membrane and the complete banjo. The match will be explored quantitatively in Section 3.2, and the reason for the choice of these particular values for mass, stiffness and dashpot impedance will be explained there. It is immediately clear that both red curves exhibit a kind of resonance, corresponding to the 


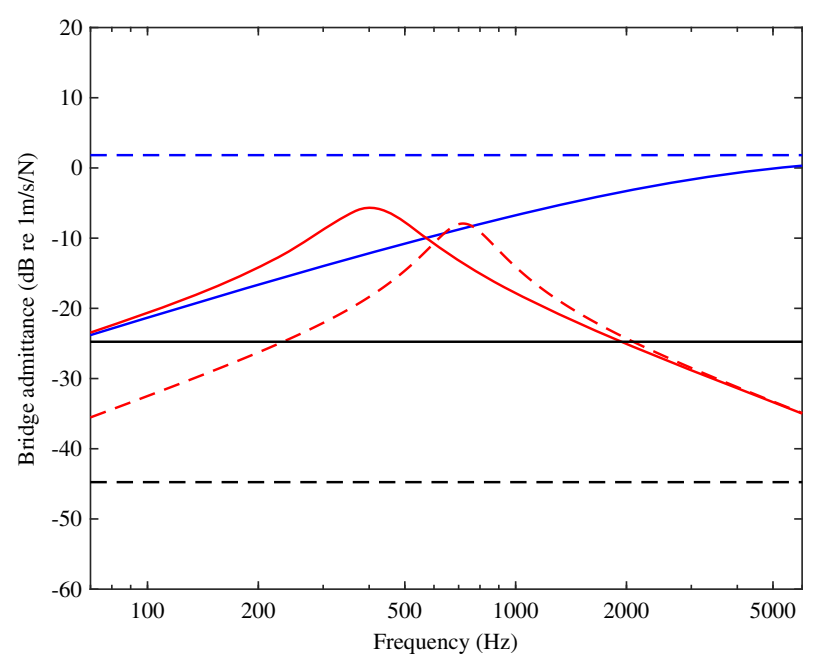

Figure 3. Estimates for the logarithmic-mean trend of admittance using Skudrzyk's method, for models related to Figure 2a. Black solid: plate with mass per unit area matching the membrane, from equation (7); black dashed: plate with mass increased by a factor 10; blue solid: membrane, from equation (8); blue dashed: asymptotic value for membrane, from equation (9); red solid: membrane with the addition of a $1.5 \mathrm{~g}$ bridge mass; red dashed: as red solid but with the addition of stiffness $20 \mathrm{kN} / \mathrm{m}$ and a dashpot of impedance $1 \mathrm{Ns} / \mathrm{m}$.

previously-mentioned formant in the envelope of the admittance of the complete banjo.

\subsection{Sound radiation and radiation damping}

The other major way in which a vibrating membrane differs qualitatively from a vibrating plate is in the nature and magnitude of coupling to the surrounding air. There are two aspects to this coupling: the kinetic energy of associated air flow produces an added-mass effect which lowers the resonance frequencies, and the radiation of sound contributes to modal damping.

For frequencies below about $1 \mathrm{kHz}$, the banjo body is sufficiently small compared to the wavelength of sound in air that the banjo can be treated as a compact sound source, and multipole arguments can be used to shed light on the behaviour (see e.g. [17, 18]). The measurements on the bare banjo head were carried out with the back fully open. This means that no net volume change is produced by any of the modes, and the far-field sound radiation will be at most dipole-like. Modes with at least one nodal diameter can be no stronger than quadrupoles.

Adding the resonator back can increase the radiation efficiency. For the simplest idealisation, treating the resonator and the rest of the banjo structure as rigid, the lowest mode of the membrane will couple to the Helmholtz resonator formed by the vented back. As shown in Figure 6 of the previous paper [1], the result is a pair of modes involving combinations of both types of motion. This behaviour is very familiar from simple models of guitar bodies [19], and also from the analysis of ducted loudspeaker enclosures (see e.g. [20]). Related effects will no doubt arise from coupling of the membrane to higher acoustic modes in the cavity. More important is the fact that axisymmetric modes of the membrane can now produce net volume change, and hence monopole radiation. Modes with nodal diameters can become dipole-like. As an aside, it may be noted that when an open-back banjo is played in the normal manner it does not behave quite like the open-back measurements here. Proximity to the player's body produces an effective vented enclosure, rather similar to the effect of the resonator back [21].

Sound-structure coupling at higher frequencies is usually discussed in terms of the behaviour of an infinite system, plus corrections to allow for the effects of finite size. As applied to sound radiation from vibrating plates, this method is highly developed and familiar. Bending waves in a plate are dispersive whereas sound waves are nondispersive, and the two dispersion curves cross at the "critical frequency" [22]. Below this, an infinite plate produces only evanescent waves in the adjacent air, but above the critical frequency efficient sound radiation can take place. Membranes, however, have been relatively little studied, and the behaviour is different (see chapter 14 of [9]). Waves on a membrane are non-dispersive, and it appears that for any realistic parameter values for a banjo or drum membrane, the wave speed is slower than the speed of sound in air [1]. This means that an infinite ideal membrane can only generate evanescent waves: there is no critical frequency, and far-field sound radiation can only occur through finite-size effects. In a real membrane there will be small but non-zero bending stiffness, resulting in a critical frequency, but this is too high in the frequency range to matter for the banjo.

All modes with dipole- or quadrupole-like behaviour involve significant local air flow, with associated kinetic energy. Similarly, the evanescent waves generated by subsonic structural motion on an infinite membrane or plate carry kinetic energy. This results in an increase in the effective mass of the membrane or plate, and the low mass of a membrane makes the effect more significant in that case. For a plane wave on an infinite plate or membrane, the kinetic energy scales proportional to the wavelength (see for example Sect. 13.3.5 of Chaigne and Kergomard [9]). Generally similar behaviour can be expected for the modes of the banjo head, so the additional mass will be greater for the lower modes with longer wavelengths. The details, however, can be complicated: within musical acoustics this has been explored in the context of the behaviour of drums. The orchestral kettledrum relies on such added mass effects to achieve the near-harmonic tuning of certain modal frequencies, necessary to produce a sound of definite pitch [23].

For sound radiation, there is a simple qualitative interpretation of finite-size effects. The lower mode shapes of the banjo membrane contain very few wavelengths, so the $2 \mathrm{D}$ Fourier transform of these shapes will spread significantly into the region of wavenumber space that exceeds the sonic threshold. This means that they can radiate sound with moderate efficiency. But higher modes involving progressively more wavelengths behave more and more like the infinite membrane, with only very weak sound radiation. 


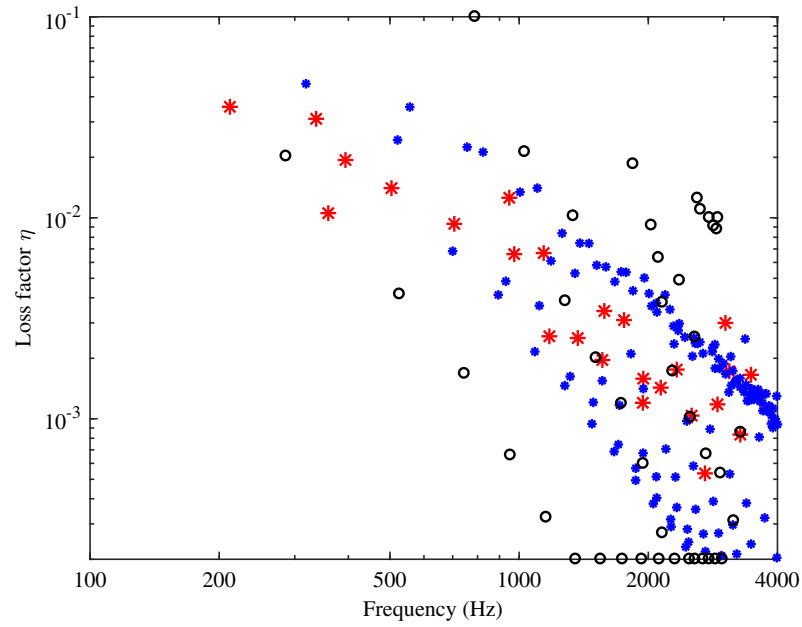

Figure 4. Loss factor against frequency for fitted modes of the bare head of the Deering banjo (red stars), compared to predictions of the square banjo model (blue stars), and the FE/BE model (black circles). Note that these particular measurements were made with the resonator back fitted to the banjo, so the fundamental frequency is significantly lower than in either model prediction.

This leads to an expectation that radiation loss factors for the membrane will decrease as frequency rises.

A quantitative prediction of this effect can be obtained for the case of sound radiation by a rectangular membrane set in an infinite plane baffle. Leppington et al. [24] have given a set of explicit approximate expressions for radiation loss factors for this case, based on asymptotic expansions assuming high wavenumber. These can be incorporated into the "square banjo" model introduced earlier. Figure 4 shows the results, as blue stars, for the same membrane model used in Figure 2. The figure also includes (as black circles) results from the $\mathrm{FE} / \mathrm{BE}$ model: discussion of these is deferred until Section 2.3.

The red stars in Figure 4 show experimental points for the bare head of the Deering banjo, measured by the procedure leading to Figures 4 and 10 of the earlier paper [1] and described in detail in previous work $[16,25]$. Both blue and red points form clouds occupying a similar region in the plot. The measured loss factors fall by more than an order of magnitude across this frequency range, reaching values of the order of $10^{-3}$ at higher frequencies. But at any given frequency there is considerable scatter in the loss factors: modes at similar frequency can have loss factors differing by up to a factor of 5 .

Previous literature (e.g. [26]) gives an explanation for the scatter of loss factors seen in Figure 4. Depending on their symmetry or anti-symmetry relative to the two central axes of the rectangular plate or membrane, modes can be divided into classes that are given labels like "edge modes" and "corner modes". These different classes have systematically different radiation damping, which (at least at low frequency) can be understood in terms of dipole-like cancellation between adjacent cells of the vibration pattern, which can account for all cells if there is an even number, but leaves one left over if there is an odd number on either or both of the axes. These classes of mode determine the obvious groupings among the blue points. The details are more complicated for the circular membrane, but a similar scatter is to be expected.

\subsection{FE/BE model of the bare membrane}

To obtain predictions that address the full detail of the banjo measurements, computations have been carried out using coupled Finite Element (FE) and Boundary Element (BE) modelling, created using the commercial vibro-acoustic software package wave ${ }^{6}[27]$. The pre-tensioned membrane was modelled with FE, coupled to air on both sides modelled with $\mathrm{BE}$. The average element size for the $\mathrm{FE}$ grid was $1.5 \mathrm{~mm}$ whereas average element size on the BE grid was $8.5 \mathrm{~mm}$. The FE model used pre-tensioned linear shell elements (a mixture of QUAD4 and TRIA3), so that the non-vanishing bending stiffness of the membrane is automatically included. FE and BE were two-way coupled to one another using conservative mapping. The wall of the pot, assumed rigid, was explicitly accounted for in the $\mathrm{BE}$ model. The resonator back was not included. The FE model was described by its in vacuo modes extracted up to $8 \mathrm{kHz}$. Convergence tests were carried out to ensure that the chosen mesh gave satisfactory performance up to this frequency.

The first step is a model of the bare membrane. A membrane with the correct thickness and the mechanical properties of Mylar was stretched over the pot. Uniform circumferential tension was applied, and adjusted until the lowest mode matched the measured frequency of the banjo, $290 \mathrm{~Hz}$. But this must be the frequency including the effect of air loading, represented in the model by boundary elements completely surrounding the banjo model to simulate free-space conditions. The resulting tension is the one listed in Table 1.

The computations reveal that in order to achieve the correct air-loaded frequency for the lowest mode, the in vacuo fundamental frequency of the membrane needs to be approximately $380 \mathrm{~Hz}$. Since the resonance frequency scales with the square root of the mass per unit area, it can be deduced that for this mode the added mass from the air loading is similar to the actual mass of the membrane. From the estimated frequency shifts of other modes below $2 \mathrm{kHz}$, the added mass can be similarly calculated. The result is plotted in Figure 5, expressed as a multiple of the mass per unit area of air within one acoustic wavelength of the surface. The scatter of points is almost horizontal, confirming the expectation that the added mass from air loading scales with the wavelength. The dashed line in this plot shows the predicted value for a plane wave on an infinite membrane in contact with air on both sides, given in terms of the sound speed in air and the in vacuo wave speed of the membrane by the formula (see [9], Sect. 13.3.5)

$$
\frac{1}{\pi \sqrt{\left(c_{\text {air }} / c\right)^{2}-1}} \approx 0.135 .
$$




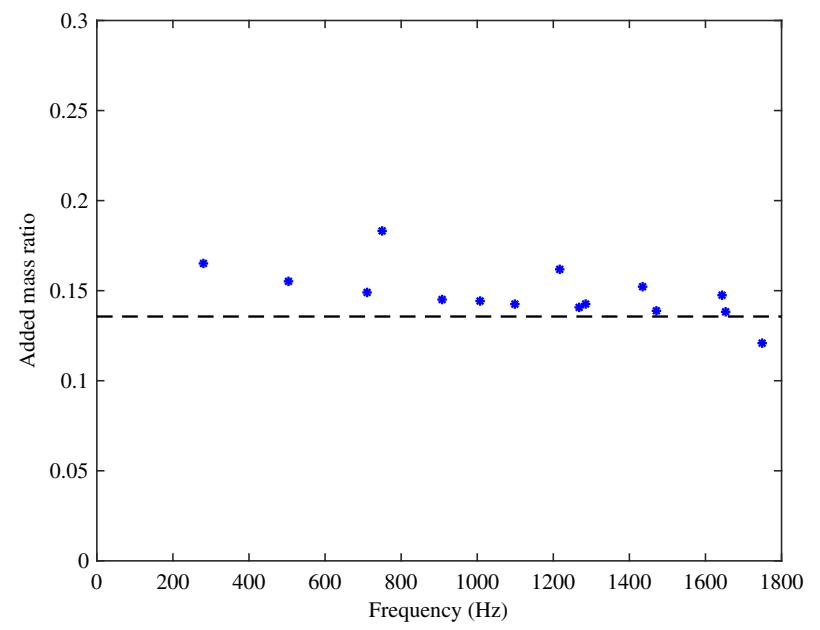

Figure 5. Added mass per unit area from air loading, expressed as a fraction of the mass per unit area of air within one acoustic wavelength, for the first 15 modal frequencies of the membrane. Dashed line: corresponding value for a plane wave of the same wavenumber on an infinite membrane.

The FE/BE model will be used to predict radiation loss factors to compare with the earlier measurements and square banjo predictions. But first, it can be used to examine the detailed differences between baffled and unbaffled cases. The FE/BE model was used to compute the point admittance, to compare with measurements such as Figure 1. For this purpose, radiation damping was included, but initially no other source of damping was added. Two cases were computed: one version with the banjo model in free space, the other with it embedded in an infinite plane baffle (evaluated using the wave ${ }^{6}$ option for two-sided SEA-SIF with a simple baffle assumption [28]). The results are shown in Figure 6a, and a direct comparison of the radiation loss factors for the two cases is shown in Figure 7. Radiation damping has been computed by dividing the input power to the BE model at each frequency by the total energy in the structure, including an allowance for the kinetic energy in the air based on equation (11). As would be expected, the unbaffled case exhibits lower radiation damping than the baffled case at lower frequencies: multipole arguments can be applied here, and the effect of a baffle on radiation efficiency will be somewhat similar to that of the resonator back, discussed above. At higher frequencies the two cases yield a broadly similar range of levels of damping.

The discrete points added to Figure 7 correspond to individual modes in the unbaffled case, obtained from the admittance shown in Figure 6a. Points plotted as stars were calculated by first taking an inverse FFT of the admittance and then processing the resulting time series in the same manner as used to generate the measured points in Figure 4. However, some peaks were not resolved clearly enough for this procedure to fit them. The circles show estimates for these missed modes: those with high damping were estimated using circle fitting, while those with damping too low to be resolved in the computed admittance were assigned a constant loss factor of $2 \times 10^{-4}$ for the purposes of this plot.
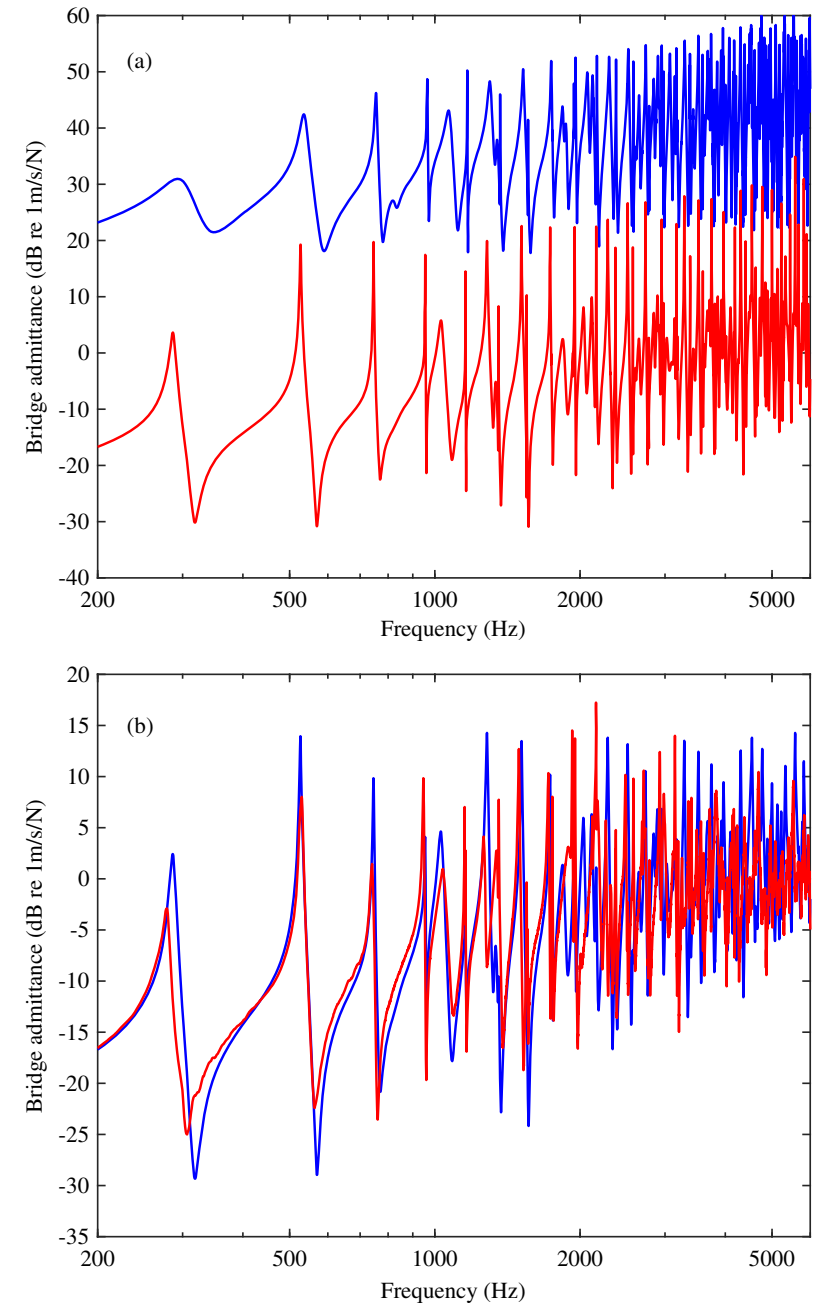

Figure 6. (a) FE/BE unbaffled prediction of point admittance of the bare membrane (red) compared to the corresponding baffled prediction (blue). The baffled result has been offset by 40 $\mathrm{dB}$ for clarity. (b) measured admittance (red) compared to FE/ BE unbaffled prediction including small structural damping (blue).

To compare these results with the measurements, the same cloud of discrete points was included in Figure 4 alongside the predictions of the "square banjo" model using the Leppington estimates for damping [24]. Referring back to that plot, both sets of model predictions show a somewhat similar distribution to the measured points, but neither matches entirely convincingly. The $\mathrm{FE} / \mathrm{BE}$ predictions spread over a wider range than the measurements, including some modes with far lower damping than the measurements at similar frequencies. The "square banjo" predictions are significantly closer to the measurements. They give a cloud with a similar spread to the measurements, but with a steeper slope in the plot: they tend to over-predict damping at low frequency, as anticipated given the assumption of a baffle, and also to under-predict it at higher frequencies.

Both theoretical models predict some loss factors lower than anything observed. But in the real banjo there are of course additional sources of energy loss that will limit these 


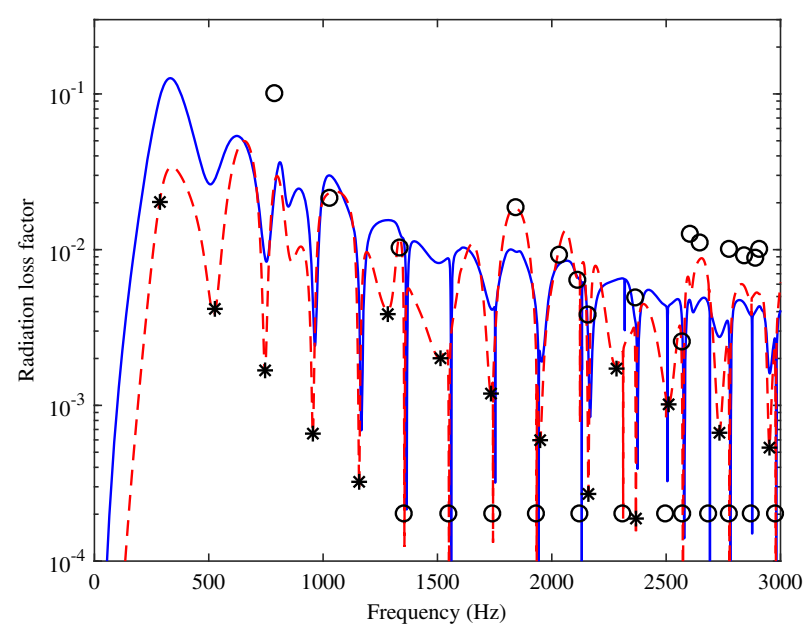

Figure 7. Computed radiation loss factor for the unbaffled case (red dashed line) and the baffled case (blue line). Discrete points indicate the frequency and loss factor of individual modes: see text for details.

very low values. As is familiar from many other problems involving the modelling of damping in mechanical vibrating systems, it is hard to give a theoretical estimate. It is not obvious what the exact mechanisms of this additional damping are: candidates include viscous dissipation associated with the non-radiating air flow around the membrane, material dissipation within the membrane, losses into the tone ring and pot through the edges of the membrane, and local friction effects around the edge of the membrane.

As a crude representation of this additional damping, a background structural damping contribution with loss factor $4 \times 10^{-3}$ was added to the $\mathrm{FE} / \mathrm{BE}$ model. In Figure $6 \mathrm{~b}$, the measured admittance is compared directly with the resulting FE/BE unbaffled prediction. The comparison is encouragingly good: many aspects of the behaviour are well captured, although it may be noted that some peak heights are significantly over-estimated. This result highlights another feature of the banjo which contrasts with the guitar, or any other wooden instrument. For many modes of the banjo membrane, radiation damping dominates over structural damping. This suggests that a high proportion of the energy put into the head of a banjo by the vibrating strings is radiated as sound. This is not the case for a plate-based instrument, where radiation damping is generally a small perturbation on the structural damping so that most of the energy from the strings is dissipated as heat in the body.

The individual points in Figure 7 show some clear patterns, and these can be associated with the corresponding mode shapes on the membrane. It is convenient to use the standard notation $(j, k)$ to denote a mode with $j$ nodal diameters and $k$ nodal circles (including the one at the edge) [23]. In these terms, the line of points starting at the fundamental mode and sloping diagonally downwards to high frequency corresponds to modes $(0,1),(1,1),(2,1),(3,1)$, etc. Recall that points shown as circles near the bottom of the plot have the correct frequency but not the correct loss factor: these points may in fact correspond to much lower values of loss factor. A second line of points starting from a circle at about $770 \mathrm{~Hz}$ and then sloping diagonally down in a similar manner corresponds to modes $(0,2)$, $(1,2),(2,2)$, etc. This pattern is presumably an equivalent for this circular problem of the "edge modes" and "corner modes" of a rectangular membrane or plate, mentioned earlier.

The pattern revealed in Figure 7 sheds some light on a piece of banjo lore. When adjusting the tension of a banjo head, it is often recommended to "tune it to a pitch of $\mathrm{G}$ or $\mathrm{G \# ".} \mathrm{It} \mathrm{might} \mathrm{be} \mathrm{imagined} \mathrm{that} \mathrm{this} \mathrm{pitch} \mathrm{refers} \mathrm{to}$ the fundamental or some particularly strong mode of the head, but this seems not to be the case. The desired pitch is most easily heard by resting a hand on the bridge to damp motion there, then tapping or rubbing the head membrane near the edge. The explanation probably lies in the pattern just shown for the modes $(0,1),(1,1),(2,1),(3,1)$, etc. At higher frequencies, these modes which have only nodal diameters become increasingly concentrated near the edge of the membrane, and their spacing is very regular. By tapping or rubbing near the edge, a mixture of these modes is excited. This results in a perceived pitch determined by the spacing of the frequencies, via the "missing fundamental" effect familiar in psychoacoustics (see e.g. Moore [29]) and relevant to many tuned percussion instruments such as the kettledrum (see e.g. [4, 23]). For the computed case illustrated in the plot, the difference between successive frequencies of these $(n, 1)$ modes is around $192 \mathrm{~Hz}$, a little lower than $G_{3}(196 \mathrm{~Hz})$. This is indeed the correct octave for the perceived pitch. This behaviour of a set of modes confined close to the edge of the membrane is an example of the acoustical phenomenon of the "whispering gallery": see for example Rayleigh [10].

It is useful to compare these results with an admittance computed using the "square banjo" model. Once this simple model can be shown to allow reasonably realistic predictions, it becomes valuable in sound synthesis: the model is fast to run and fully parametric, so it gives great flexibility for exploration of the effect of structural modifications. Figure 8 shows a case directly comparable to Figure $6 \mathrm{~b}$. The model used in Figure 2 has been enhanced by including an empirical version of the added mass from air loading, by using the fixed value 0.15 to approximate the nearly horizontal line shown in Figure 5 for the modes at lower frequency. It incorporates the baffled radiation damping, together with a background level of structural damping with value $4 \times 10^{-3}$, exactly as in the $\mathrm{FE} / \mathrm{BE}$ comparison of Figure $6 \mathrm{~b}$.

The detailed results do not, of course, match the measurement as accurately as those of Figure $6 \mathrm{~b}$ because the geometry is different. Peak frequencies are reduced by the added-mass approximation, but the lowest mode still lies higher in frequency than the measured value because a circular membrane is known to give the lowest frequency of any membrane of a given area. The second and third peaks in the measured results both appear as pairs of peaks in the model predictions because the degeneracy of the circular case has been deliberately broken by the choice of 


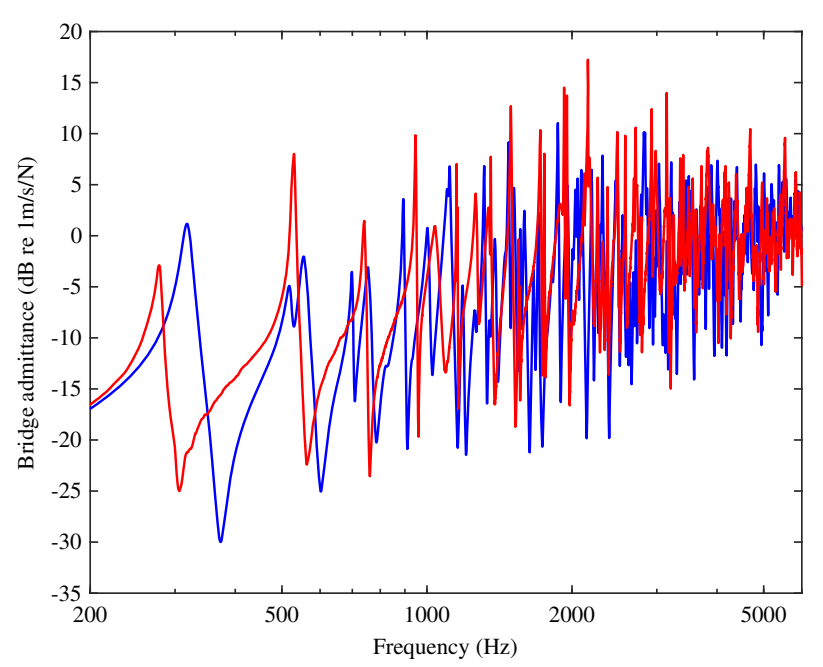

Figure 8. Bridge admittance, measured (red) compared to the "square banjo" model as in Figure 2 but using the empirical added-mass model, and the radiation damping model together with assumed background structural damping $4 \times 10^{-3}$.

rectangular membrane dimensions. However, at higher frequencies the general match is encouragingly good.

\subsection{Static compliance and the membrane singularity}

Before leaving the topic of the behaviour of the bare banjo membrane, it is useful to investigate a consequence of the singularity mentioned earlier. In modal terms, equation (5) shows that below the lowest modal frequency,

$$
Y\left(x_{b}, y_{b}, \omega\right) \approx i \omega \sum_{n, m} \frac{u_{n m}^{2}\left(x_{b}, y_{b}\right)}{\omega_{n m}^{2}}=i \omega C,
$$

where $C$ is the static compliance at the chosen point. The amplitude factor $u_{n m}^{2}\left(x_{b}, y_{b}\right)$ is statistically constant for a single point $\left(x_{b}, y_{b}\right)$, for any uniform system [12]. For a plate-like system, the $N$ th natural frequency varies approximately proportional to $N$, so that the sum converges quickly and the static compliance is dominated by the low modes. However, the dispersion law of the membrane means that the $N$ th natural frequency varies approximately proportional to $\sqrt{N}$, so

$$
C \sim \sum_{N} \frac{1}{N}
$$

For an ideal membrane and a point force, this sum would diverge logarithmically: $C \rightarrow \infty$. However, in reality something serves to make the term finite. In a simulation model of any kind, the value of $C$ will be determined by truncation of mode count or element size. For both the "square banjo" model and the finite element model presented in Section 2.3, modal truncation is the limiting factor. Such truncation is not determined by physical principles, except to be adequate to cover the desired frequency range. In the context of structural dynamics it is very unusual to find such truncation having an important influence at low frequency as seen here: it only arises because of the logarithmic divergence in the idealised membrane problem. The effect is, however, familiar in other contexts: an analogous divergence often arises in relativistic quantum mechanics.

For the measured response of the real banjo, bending stiffness, finite footprint of the force and local nonlinearity near the driven point are all candidates for influencing the value of $C$, and there may be others. Pragmatically, $C$ could be regarded as a constant that is not well determined by theoretical modelling, although it is readily deduced from a measurement of admittance. This resolution is rather like the renormalization program for quantum electrodynamics, first established by Feynman, Schwinger, and Tomonaga (see e.g. [30]). The divergent effects of very short wavelengths at first appear to have an impact on virtually every physical quantity. However, if explicit questions about those short lengths are avoided, their impact on everything else can be absorbed into a small number of parameters that are deduced from actual measurements.

It is quite straightforward to measure the static compliance directly. A weight can be placed on the membrane through a footprint of the desired size, and an engineer's dial gauge used to measure the deflection at the centre of the footprint. For the simplest case of a rigid circular "indenter" of radius $r$ at the centre of an ideal membrane of radius $a$ and tension $T$, the compliance is readily shown to be (see e.g. [13])

$$
C(r)=-\frac{\ln (r / a)}{2 \pi T} .
$$

Figure 9 shows the prediction of this equation using the tension listed in Table 1, compared to experimental results on the Deering head with a range of indenter sizes. The measured values follow the theoretical line accurately until the indenter is less than about $5 \mathrm{~mm}$ in diameter, when the compliance falls below the line. This confirms the accuracy of the tension, which was inferred by an independent route from the $\mathrm{FE} / \mathrm{BE}$ modelling. Bending stiffness in the head will result in an effective diameter which is larger than the physical footprint, leading to a levelling-off of the curve in the way seen here. There may also be other contributory factors.

The plot also shows the value of $C$ estimated from the measured admittance, using the fact that $Y \rightarrow \mathrm{i} \omega C$ for frequencies well below the lowest resonance. This measurement was obtained using a hammer with a contact size of the order of $1 \mathrm{~mm}$. It is hard to know exactly where to place this point on the horizontal axis, but the deduced value of $C$ is of the same order as the limiting value from the static tests, although a little lower than expected. It is worth mentioning a relevant observation about the measurement of the point admittance on the bare membrane. When this measurement was first carried out, the small impulse hammer was positioned alongside the laser spot, on the same side of the membrane. This separation of about $5 \mathrm{~mm}$ would not affect the results on most structures, but alerted by this issue of the footprint and the static response, the measurement was repeated with the hammer and laser on opposite sides so that they could be lined up more 


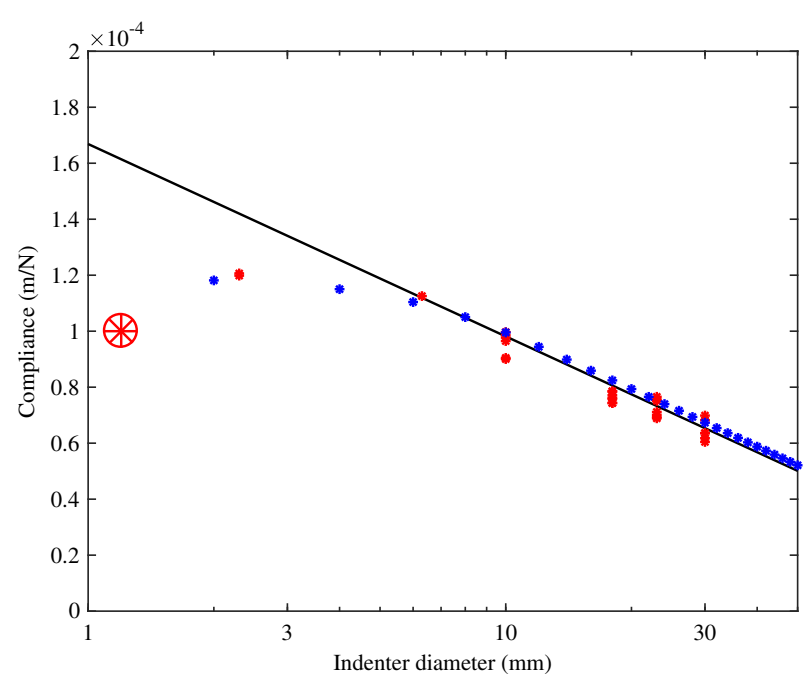

Figure 9. Small red symbols: measured static compliance at the centre of the head of the Deering banjo, for different diameters of rigid indenter. Solid line is the prediction from ideal membrane theory, using the membrane tension listed in Table 1. Large red symbol: value of DC compliance deduced from the measured admittance using a hammer with a very small area of contact. Blue symbols. values deduced for a square loading region at the centre of a square membrane, with a cutoff frequency $10 \mathrm{kHz}$.

precisely. The result was that the estimated static compliance rose by some $40 \%$.

Figure 9 also includes points obtained from the "square banjo" model. To give the closest comparison to the circular case, a square membrane was assumed, and a square loading footprint was applied at the centre. The modal sum was truncated at $10 \mathrm{kHz}$, which produced results in excellent agreement with the banjo tests. In this case the limit on wavelength produces the levelling-off of compliance.

\section{Modelling the complete banjo}

\subsection{Validation with a one-string bridge}

When the bridge and strings are added to the bare membrane, it has already been seen in Figure 1a that the admittance changes very significantly. There are several possible effects operating. These include: the bridge mass and footprint, extra stiffness contributed by the string tension (both directly and by increasing the membrane tension somewhat) and by the strings' axial stiffness through the effect of the break angle over the bridge, extra damping from the fact that the strings were deliberately damped during the measurement of admittance, possible extra damping from micro-scale frictional effects at interfaces between strings and bridge or between bridge feet and membrane. Furthermore, the bridge itself may exhibit resonant behaviour.

The bridge of the Deering banjo has a rather complicated three-legged shape: see Figure 1 of the earlier paper [1]. Before trying to model this (to be discussed in Sect. 3.3), a simpler case is studied first in which effects of the bridge and strings are modelled as a single-"point" constraint on the membrane (but using a finite footprint to avoid the singularity). Bridge mass, stiffness from the strings and added damping can all be represented this way, as has already been illustrated via Skudrzyk's method in Figure 3 . It is useful to see how far such a model can be pushed: note that the point coupling result in equation (10) can be applied to the full admittance just as well as to the infinitemembrane admittance: simply ignore the $\infty$ symbols so that it relates $Y(\omega)$ to $Y_{b}(\omega)$.

Experimental comparisons for this approach have been obtained. A solid circular cylinder of wood of diameter $12.7 \mathrm{~mm}$ was shaped to make a "one-string bridge", and fitted to the banjo using just the middle (3rd) string. The height of this bridge was such that the break angle of the string over the bridge was approximately the same as on the regular bridge. Measurements were made with this bridge, of mass $1.35 \mathrm{~g}$, placed $85 \mathrm{~mm}$ from the edge, the same distance as the bare-membrane admittance shown in Figure 1a.

Results are shown in Figure 10, compared to corresponding predictions from the $\mathrm{FE} / \mathrm{BE}$ model with a rigid cylinder of the correct diameter and mass attached to the membrane. Figure 10a shows the comparison of admittances of the bare membrane, while Figure 10b shows the corresponding result after adding the bridge. The blue curve in Figure 10b shows the result of using equation (10) to add the bridge mass, based on the measured admittance from Figure 10a.

The level of agreement revealed by these plots gives some confidence in the basic accuracy of the computer model, and also in the calibration accuracy of the measurements. However, there are anomalies. The lowest one or two peaks are consistently predicted too high in amplitude, typically by $10-15 \mathrm{~dB}$, even though the frequencies and damping factors are generally well matched by the $\mathrm{FE} / \mathrm{BE}$ calculations. The match with the point-coupling calculation is generally close, but even this predicts an anomalously high second peak. This makes the apparently simple circular membrane very unusual among vibration problems. Normally, the low modes are the easiest to match with model predictions, while higher modes become progressively less accurate. But here the converse seems to be the case. An important ingredient of the underlying physics will be explored in Section 3.4.

\subsection{Shaping the formant}

Having seen that the point-coupling method gives good results for adding the simple cylindrical bridge to the membrane, it is natural to try the same approach for predicting the admittance with the real banjo bridge. Figure 11a shows a comparison between the measured response of the complete banjo (in red) and the point-coupling prediction using a concentrated mass of $2.2 \mathrm{~g}$ to represent the bridge, and using the measured admittance of the bare membrane from Figure 1 for $Y\left(x_{b}, y_{b}, \omega\right)$ (blue solid line). The low-pass filtering effect of the bridge is captured, at least qualitatively. However, the detailed match is disappointingly poor 

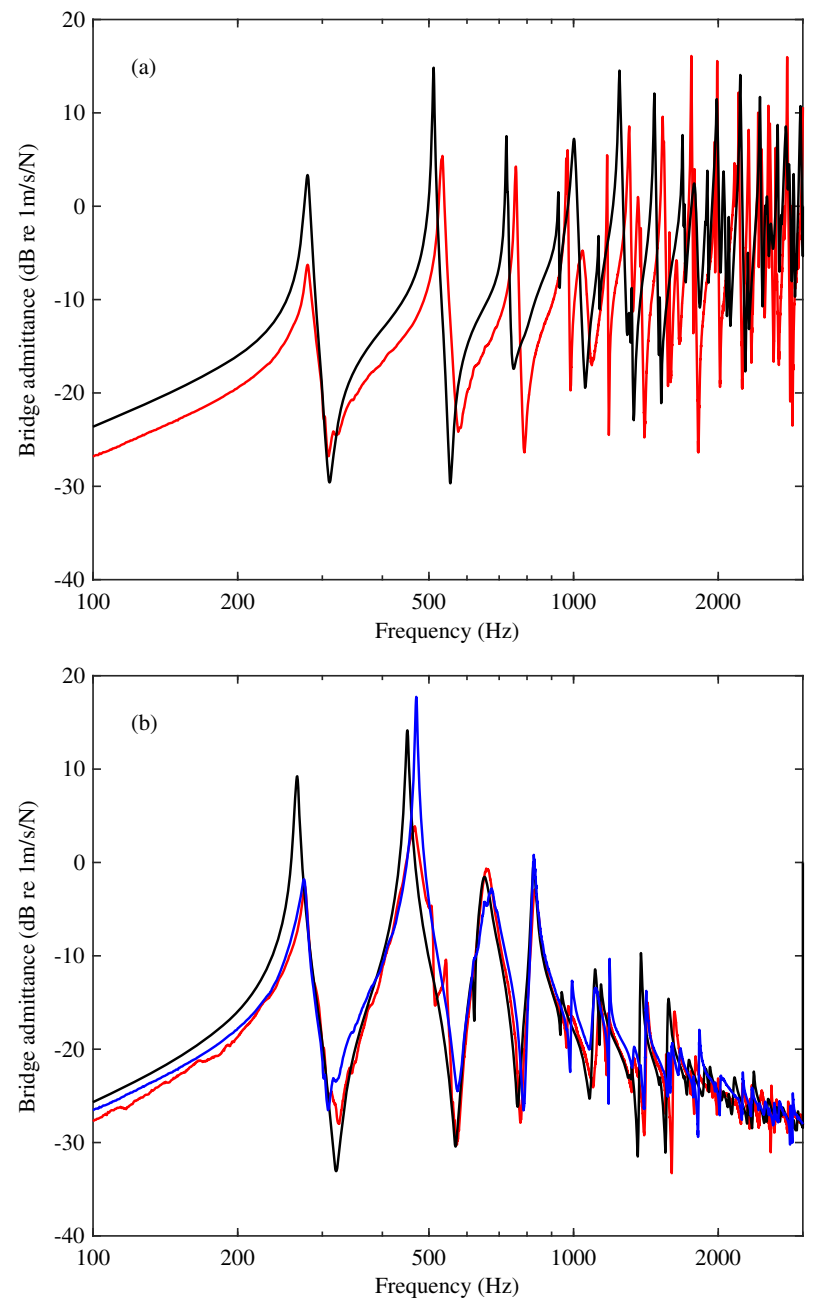

Figure 10. Admittance results for the banjo with a rigid circular bridge: (a) bare membrane; (b) bridge-loaded membrane. Red curves: measured; black curves: predicted by FE/BE model; blue curve: result of using the point-coupling formula (10) with the bridge mass and the measured bare-membrane admittance.

after the success seen in Figure 10: neither the pattern of peak heights nor the range of fluctuations is well reproduced. The first and second resonance peaks are too low in frequency, and the first is too high in amplitude by almost $20 \mathrm{~dB}$.

Figure 11a also shows the effect of two alternative masses for the "bridge": $1.5 \mathrm{~g}$ (dashed line) and $1 \mathrm{~g}$ (dashdot line). Varying the mass makes no difference at the lowest frequency, but it has very strong effects on the peak frequencies and heights up to about $800 \mathrm{~Hz}$. At frequencies above about $1 \mathrm{kHz}$, the effect is more clear-cut: the response is dominated by the mass as would be expected, and the three curves follow parallel tracks. In the range $1-3 \mathrm{kHz}$, the curve corresponding to mass $1.5 \mathrm{~g}$ matches the measured response best. Around $3 \mathrm{kHz}$, the measured admittance jumps up whereas the predicted curves continue to fall. This feature will be explained in Section 3.3.
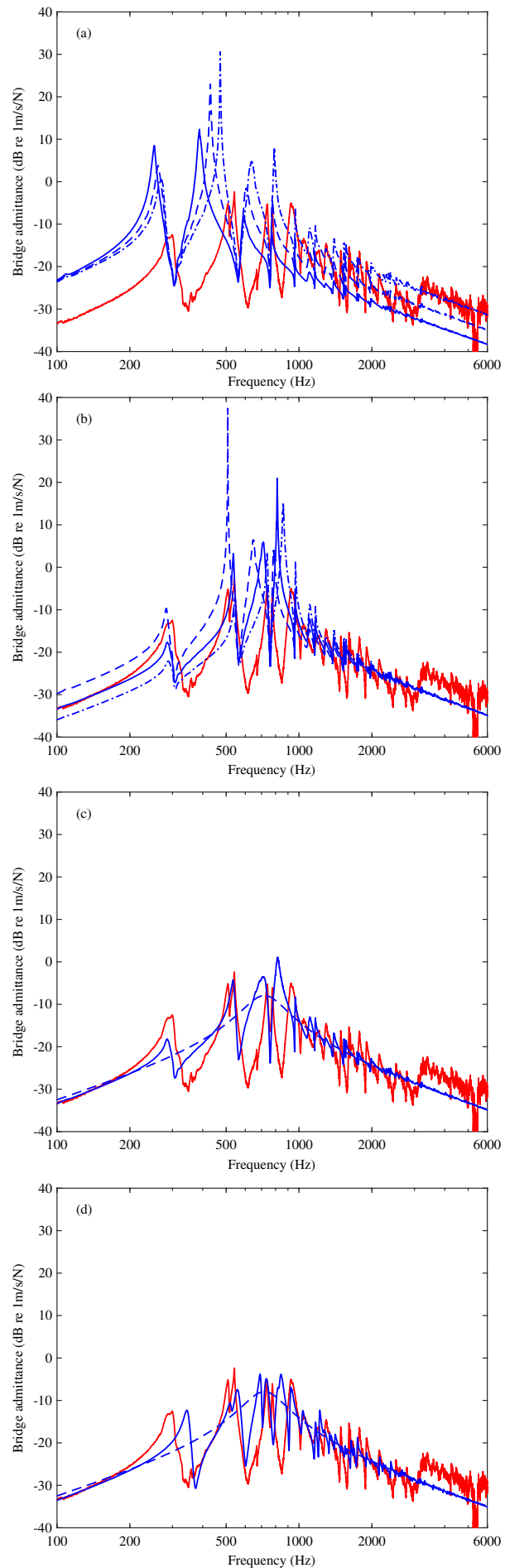

Figure 11. Measured bridge admittance of the Deering banjo with bridge and damped strings but without resonator (red), identical in (a)-(d), compared to (a) result of adding concentrated bridge masses to the measured admittance of bare head: $2.2 \mathrm{~g}$ (blue solid), $1.5 \mathrm{~g}$ (dashed); $1 \mathrm{~g}$ (dash-dot); (b) as solid blue line from (a), but adding stiffness $k_{s}$ equal to $20 \mathrm{kN} / \mathrm{m}$ (blue solid), $10 \mathrm{kN} / \mathrm{m}$ (blue dashed) and $30 \mathrm{kN} / \mathrm{m}$ (blue dash-dot); (c) as solid blue line of (b) but adding a viscous damper of strength $1 \mathrm{Ns} / \mathrm{m}$; (d) bridge model as (c), but added to admittance from the square banjo model. Dashed blue lines in (c) and (d): Skudrzyk mean-value prediction as in Figure 3. 
The next effect to be included is an additional stiffness acting at the bridge, which can be added using equation (10) with an effective stiffness $k_{s}$. One contribution to this stiffness arisies from axial stiffness of the strings, and can be estimated by assuming that the small axial stretch of the strings in response to motion at the bridge is distributed uniformly along their entire length, on both sides of the bridge. The result is

$$
k_{s}^{(1)} \approx \frac{E A \sin ^{2} \beta}{a+L},
$$

where $L$ is the vibrating string length, $a$ is the afterlength, $A$ is the combined cross-sectional area of the steel strings, $E$ is the Young's modulus of steel, and the break angle over the bridge is $\beta$. The resulting value of the combined stiffness of the five strings, based on their diameters and a break angle of $13^{\circ}$, is approximately $5 \mathrm{kN} / \mathrm{m}$. A closely related stiffening effect will arise from the deformation of the head under the static load of the strings, which will increase the net stiffness. The membrane beneath the bridge feet will show a kind of "break angle" similar to the strings across the top of the bridge, but the details are more complicated to analyse than for the strings.

Another contribution to stiffness is associated with the tension of the strings, again mediated by the break angle. To obtain an estimate, the bridge can be assumed to move perpendicular to the main vibrating length of string, so that this extra stiffness comes entirely from the afterlengths. The result is

$$
k_{s}^{(2)} \approx \frac{T_{\mathrm{tot}} \cos ^{2} \beta}{a},
$$

where $T_{\text {tot }}$ is the total tension of the five strings. The numerical result is approximately $2.4 \mathrm{kN} / \mathrm{m}$.

In the light of uncertainty over the precise value of $k_{s}$, an empirical approach can be followed. Figure 11b shows the result of adding the effect of three alternative values of stiffness to the dashed curve in Figure 11a; the case with mass $1.5 \mathrm{~g}$ that gave the best match to the high-frequency trend. It is clear that stiffness has a significant effect. The results show an opposite pattern to those in Figure 11a: as would be expected, changing the stiffness has no effect at high frequency, but a strong and systematic effect at the lowest frequencies. The solid curve, with $k_{s}=20 \mathrm{kN} / \mathrm{m}$, gives the best match to the measurements below about $200 \mathrm{~Hz}$. The addition of stiffness has raised the lowest resonance frequencies to roughly the correct places. Similar to what was seen in Figure 11a, the peak heights below about $1 \mathrm{kHz}$ are very sensitive to the value of stiffness. The physics behind this sensitivity will be explored in Section 3.4.

Figure 11c shows the effect of adding some additional damping to Figure 11b. The change is entirely ad hoc, using a value $\lambda=1 \mathrm{Ns} / \mathrm{m}$ in equation (10). The blue dashed curve shows the corresponding Skudrzyk mean-value estimate. For frequencies below $3 \mathrm{kHz}$, the qualitative match to measurements is now good. What has been achieved by these steps of choosing a mass to match the high-frequency trend and a stiffness to match the low-frequency trend has been to place the formant in very much the right place to match the measured response, with the final adjustment of some added damping to keep the maximum peak height close to the observed values.

Finally, Figure 11d shows the result of adding the same single-point bridge model to the admittance predicted by the square banjo model, as seen in Figure 8. The match to the measured response seems as good as was obtained using the measured bare-membrane admittance. This is the datum version of the square banjo model that was used in the earlier paper [1] in order to create sound examples of parametric variations in the banjo structure.

Skudrzyk's method can be used to explore the behaviour of the low-frequency formant in more detail. From equation (8), the admittance $Y_{\infty}(a, \omega)$ varies slowly with frequency, so equation (10) suggests that there will be a single pole of $Y_{b}, \infty(a, \omega)$, corresponding to a zero of the right-hand side of the equation, somewhere near $\omega=\sqrt{k_{s} / m_{b}}$. A simple iterative calculation allows the complex pole frequency to be computed from that starting approximation. This pole defines the formant: the centre frequency and half-power bandwidth can be calculated from its real and imaginary parts. Figure 12 shows some examples, for a range of different values of $m_{b}$ (indicated by different colours) and $k_{s}$ (indicated by different symbols). The datum case is marked by a black circle. The specific values chosen are the same ones used on the accompanying website [4] in sound examples for varying mass and stiffness. The trends resulting from variation of $m_{b}$ and $k_{s}$ are different: decreasing mass or increasing stiffness naturally both result in increasing the centre frequency, but the bandwidth increases in one case and decreases in the other case.

\subsection{Realistic bridge model and bridge hills}

To investigate the feature seen around $3 \mathrm{kHz}$ in Figure 11, the FE/BE model was further developed to include a detailed representation of the bridge. The model was also extended to include a representation of the effect of $k_{s}$ just discussed. Figure 13a shows the measured admittance compared to the prediction of this enhanced model. Figure 13b shows the corresponding comparison for admittance measured and simulated in the lateral direction near the top of the bridge saddle. In both cases, the FE/BE results agree very well with many aspects of the measurements, especially at higher frequencies.

At low frequencies, a consistent tendency to overpredict peak heights was seen when the stiffness effect was not included. However, adding stiffness with the chosen value has given much closer agreement. This sensitivity of low-frequency behaviour to the effective stiffness from the strings and head is reminiscent of the behaviour seen in Figure 11: further discussion of this sensitivity is deferred to Section 3.4. The remaining disparities between measurement and prediction seen in Figure 13 are mostly associated with a tendency to underestimate the damping. No doubt it would be possible to make ad hoc adjustments to the damping to achieve a better match, but in the absence of a physics-based model for damping there seems little point in such an exercise. 


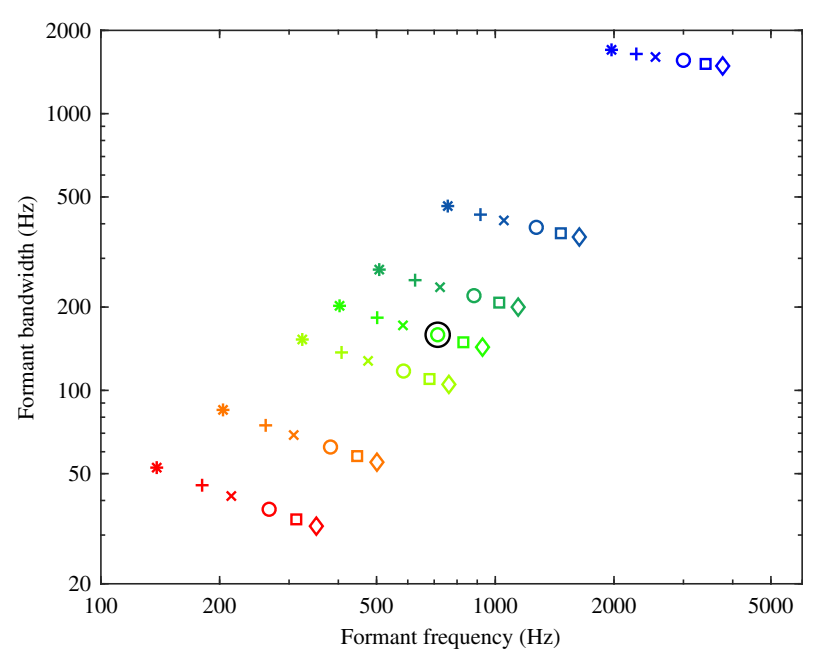

Figure 12. The centre frequency and half-power bandwidth of the low-frequency formant, fitted by Skudrzyk's mean-value method. Different masses $m_{b}=[10,5,2.2,1.5,1,0.5,0.1] \mathrm{g}$ are indicated by colours, from red to blue. Different stiffnesses $k_{s}=[0.01,5,10,20,30,40] \mathrm{kN} / \mathrm{m}$ are indicated by symbols, from stars to diamonds. The datum case is indicated by a black circle.

The feature around $3 \mathrm{kHz}$ is strikingly prominent in the lateral admittance shown in Figure 13b. The FE/BE results contain a feature (indicated by a vertical dashed line in the plot) which strongly resembles the measured feature, including the fact that it is more prominent in the lateral admittance. This feature is reminiscent of the "bridge hill" on the violin $[6,7]$. However, it is not the only "hill" evident in the behaviour of the banjo. Figure 13c shows the bridge admittance measured vertically near the third string notch, at the bridge centre. There is virtually no trace of the $3 \mathrm{kHz}$ feature, but there is a strong feature near $5 \mathrm{kHz}$. Corresponding results from the FE/BE model are included. The level of agreement with the measurements is good: the $5 \mathrm{kHz}$ "hill" is captured very convincingly, again indicated in the plot by a vertical dashed line.

To find out what is causing the observed features, images from the FE/BE simulations can be used. Figure 14 shows the pattern of forced displacement in the membrane and bridge at the two frequencies marked by vertical dashed lines in Figure 13. They both suggest the involvement of specific deformations of the bridge and membrane: both seem closely analogous to the "bridge hill" in the violin, which is caused by a combination of rocking of the violin bridge about its narrow waist, and local deformation of the top plate in the "island" area between the f-holes $[6,7]$. The banjo behaviour near $3 \mathrm{kHz}$, seen in Figure 14a, involves rotation of the three legs of the bridge. This results in some rocking of the bridge top, together with significant lateral motion at the bridge corners, which accounts for the strong lateral response. The behaviour near $5 \mathrm{kHz}$, seen in Figure 14b, involves the first bending resonance of the bridge, in the plane perpendicular to the membrane. This resonance in the free bridge falls near $2.5 \mathrm{kHz}$, but the effect of coupling to the membrane has pushed the peak frequency up significantly.
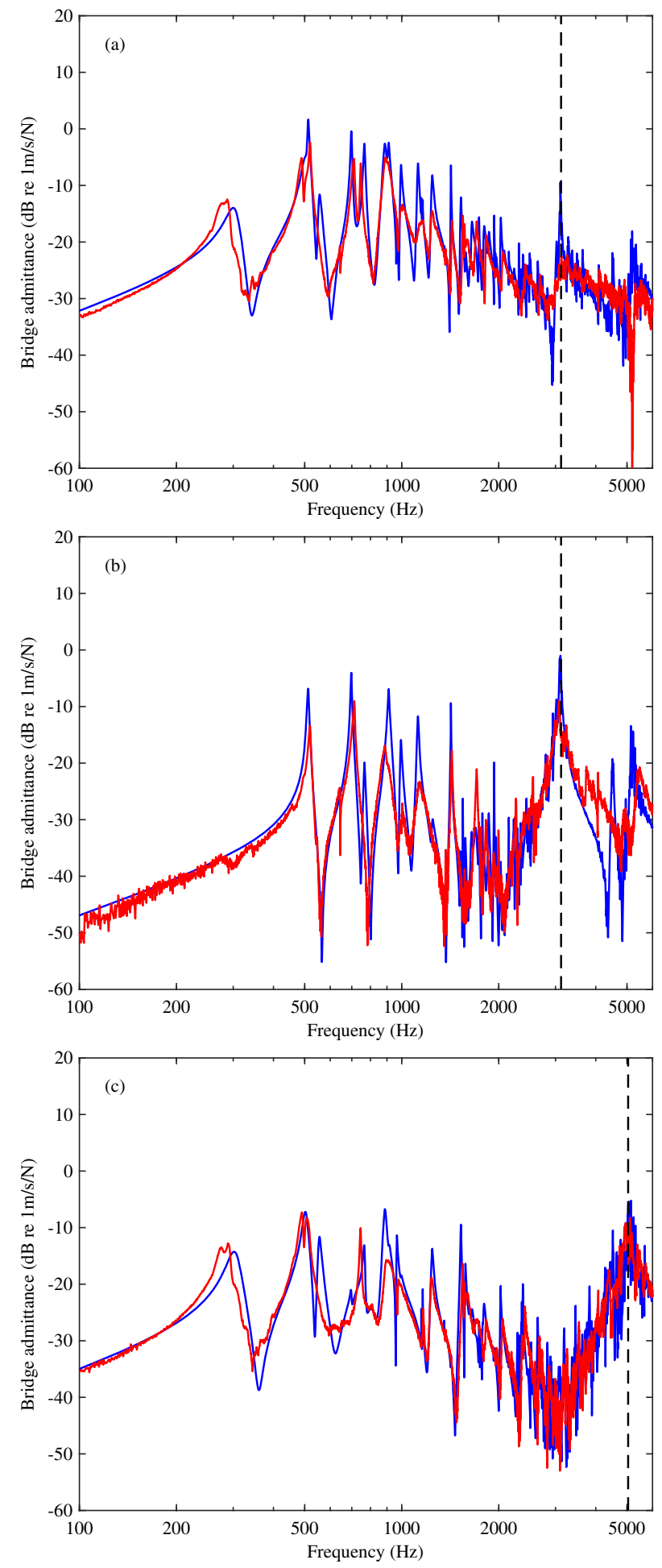

Figure 13. Bridge admittances (a) vertical at the bridge corner as measured on the Deering banjo (without resonator) (red) and as computed by the FE/BE model (blue); (b) horizontal at the bridge corner as measured (red) and as computed by the FE/BE model (blue); (c) vertical at the third string notch as measured (red) and as computed by the FE/BE model (blue). The vertical dashed lines in (a) and (b) indicate the frequency of the displacement plot in Figure 14a. The vertical dashed line in (c) indicates the frequency of the displacement plot in Figure 14b. 


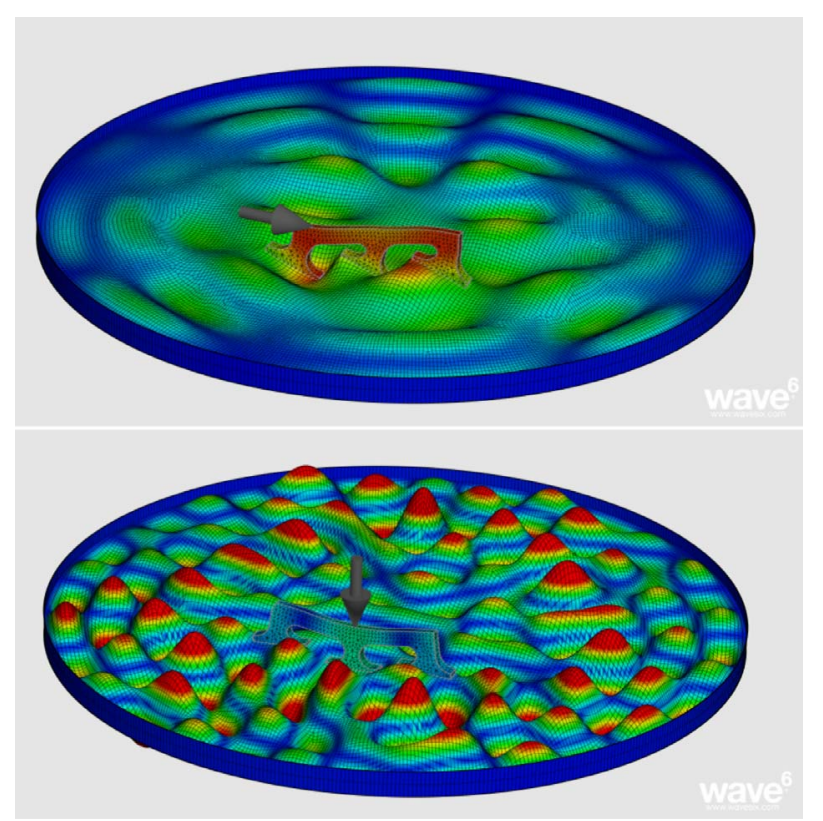

Figure 14. Displacement of membrane and bridge predicted by the $\mathrm{FE} / \mathrm{BE}$ model at the frequencies marked by vertical black lines in (a) Figure 13b; (b) Figure 13c.

In both cases, the interplay of bridge dynamics with the motion of the membrane is strongly reminiscent of the violin bridge hill. In the case of the violin the hill has been convincingly implicated in important perceptual effects, and modification of the hill's details is a key part of "tonal adjustment" carried out by instrument makers. Something similar is probably true for the banjo. The details of the two hill features will be sensitive to the design, constructional details and material of the bridge. Bridge designs on the banjo vary over a wider range than that of violin bridges, although the "3-foot" design used here is the most common in contemporary banjos. Some, with more wood near the bottom, are considerably stiffer for the same weight and outer foot span. Two-foot bridges, on the other hand, may be more flexible. It may be noted that Rae [31] reported a "formant" in this frequency range, discerned in spectral analysis of recordings of played sounds on various banjos. He advanced no physical explanation, but he probably saw a manifestation of one or both of the "hill" features discussed here.

\subsection{Low-frequency sensitivity of banjo admittance}

The sensitive behaviour at low frequency revealed in Figure 11 has been shown to relate to the formant revealed by Skudrzyk's method. A different perspective can be given by a simple modal model, allowing only for the first two resonances of the membrane plus the mass and stiffness effects at the single-point bridge. Up to the range of the second mode, where some of the sensitive dependence was seen in Figure 11, the admittance of the bare membrane should be well approximated by the form

$$
Y(\omega)=\mathrm{i} \omega C^{\prime}+\frac{\mathrm{i} \omega}{m_{1}\left(\omega_{1}^{2}+\frac{\mathrm{i} \omega \omega_{1}}{Q_{1}}-\omega^{2}\right)}+\frac{\mathrm{i} \omega}{m_{2}\left(\omega_{2}^{2}+\frac{\mathrm{i} \omega \omega_{2}}{Q_{2}}-\omega^{2}\right)},
$$

where mode $j$ has frequency $\omega_{j}$, effective mass $m_{j}$ and $\mathrm{Q}$ factor $Q_{j}$. The first term, with coefficient $C^{\prime}$, describes the net below-resonance contribution of all the higher modes. $C^{\prime}$ is related in a simple way to the static compliance of the membrane introduced earlier:

$$
C^{\prime}=C-\frac{m_{1}}{\omega_{1}^{2}}-\frac{m_{2}}{\omega_{2}^{2}}
$$

because the contributions of the first two modes are included explicitly in equation (17). As $\omega \rightarrow 0, Y$ tends towards a pure compliance as in equation (12).

The poles of $Y_{b}$, the admittance with the "bridge" added, can be found as the zeroes of $1 / Y_{b}$ from equation (10). They must therefore satisfy

$$
Y\left(x_{b}, y_{b}, \omega\right)=-\frac{\mathrm{i} \omega}{k_{s}-\omega^{2} m_{b}} .
$$

Substituting the expression (17), the poles of the reducedorder model must satisfy

$$
\begin{aligned}
& \frac{1}{m_{1}\left(\omega_{1}^{2}+\mathrm{i} \omega \omega_{1} / Q_{1}-\omega^{2}\right)}+\frac{1}{m_{2}\left(\omega_{2}^{2}+\mathrm{i} \omega \omega_{2} / Q_{2}-\omega^{2}\right)} \\
& =-C^{\prime}-\frac{1}{k_{s}-\omega^{2} m_{b}} .
\end{aligned}
$$

For the undamped case, this equation can be represented in graphical form. If the left-hand and right-hand side functions are separately plotted, the intersections will give the roots. A typical example is shown in Figure 15. It uses modal parameters fitted to the first two resonances of the measured bare-membrane admittance from Figure 1a, together with the measured value $C^{\prime}=10^{-4} \mathrm{~m} / \mathrm{N}$ and the nominal values $m_{b}=2.2 \mathrm{~g}$ and $k_{s}=20 \mathrm{kN} / \mathrm{m}$.

This graphical representation separates the effects of the original system, appearing in the blue curve, from those of the static compliance, added mass and added stiffness, all combined in the red curve. The blue curve could, of course, be extended to include more resonances of the bare membrane. The red curve is characterised by two frequencies: it goes to infinity at the frequency $\omega_{b}=\sqrt{k_{s} / m_{b}}$, and it crosses the zero line at a frequency $\Omega$ given by

$$
\Omega^{2}=\frac{k_{s}+1 / C^{\prime}}{m_{b}} .
$$

It is straightforward to visualise the effects of varying the static compliance, added mass or added stiffness. For frequencies above $\omega_{b}$, the red curve will cross the blue curve once between each pair of bare-membrane resonances. If $C^{\prime}$ is small, as it would be for a plate-based instrument like a guitar, $\Omega$ will be very large, so that well above the frequency $\omega_{\mathrm{b}}$ these crossings are well spaced out, and each crossing is a little below the original resonance. But for the case plotted, $\Omega$ falls close to the second bare-membrane 


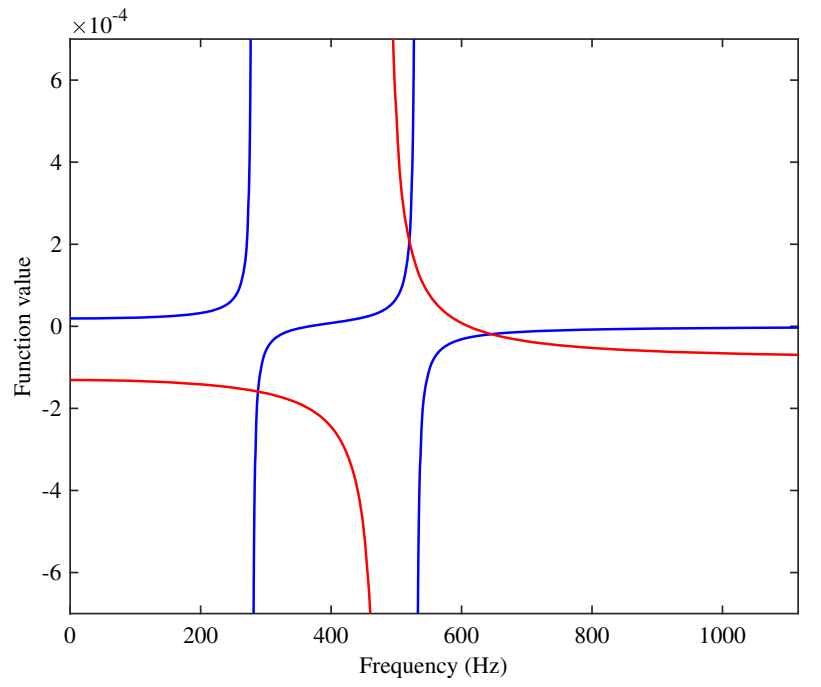

Figure 15. Graphical construction for the roots of equation (20).

resonance, and this results in a pair of crossings relatively close in frequency. Furthermore, for this case $\omega_{b}$ lies above $\omega_{1}$, with the result that the first resonance frequency is raised rather than lowered, just as was seen with the real banjo bridge.

To see what this means, it is necessary to include damping. Numerical results are then needed. Equation (20) leads to a sixth-order polynomial in $i \omega$ with real coefficients, the roots of which can be found using the Matlab routine roots. The influence of any chosen parametric variation can then be investigated by computing a root-locus plot. Three examples are shown in Figure 16. The nominal case uses the fitted modal parameters and the same values of $C, m_{b}$ and $k_{s}$ as for Figure 15. Plots have been generated in which $C, m_{b}$ and $k_{s}$ are varied in turn, each ranging from zero up to three times its nominal value, while keeping everything else fixed. In each plot, the horizontal axis shows the real part of the pole frequency, while the vertical axis shows the loss factor associated with each pole. The poles are indicated by lines of red stars, and the end of the line corresponding to the value zero is marked with a circle. The two original membrane poles are shown as large green symbols, and the row of green stars at the bottom of each plot indicates the relevant range of the parameter $\Omega$. The roots for the nominal case are indicated by black stars: they can be seen to fall at frequencies matching the intersections seen in Figure 15.

Figure 16 a shows the effect of varying $C^{\prime}$. When $C^{\prime}$ is very small, two poles lie near to the original pair, while the third is out the picture at very high frequency. As $C^{\prime}$ increases, all three pole frequencies move down as would be expected with decreasing stiffness. The parameter $\Omega$ moves down past $\omega_{2}$ but does not reach $\omega_{1}$ in the range studied. The result is a strong interaction between the two highfrequency poles, with the higher one ending close to the original second pole. The middle and lowest poles move down: if the range of $C^{\prime}$ were extended to higher values, the middle pole would eventually approach the original pole. The lowest
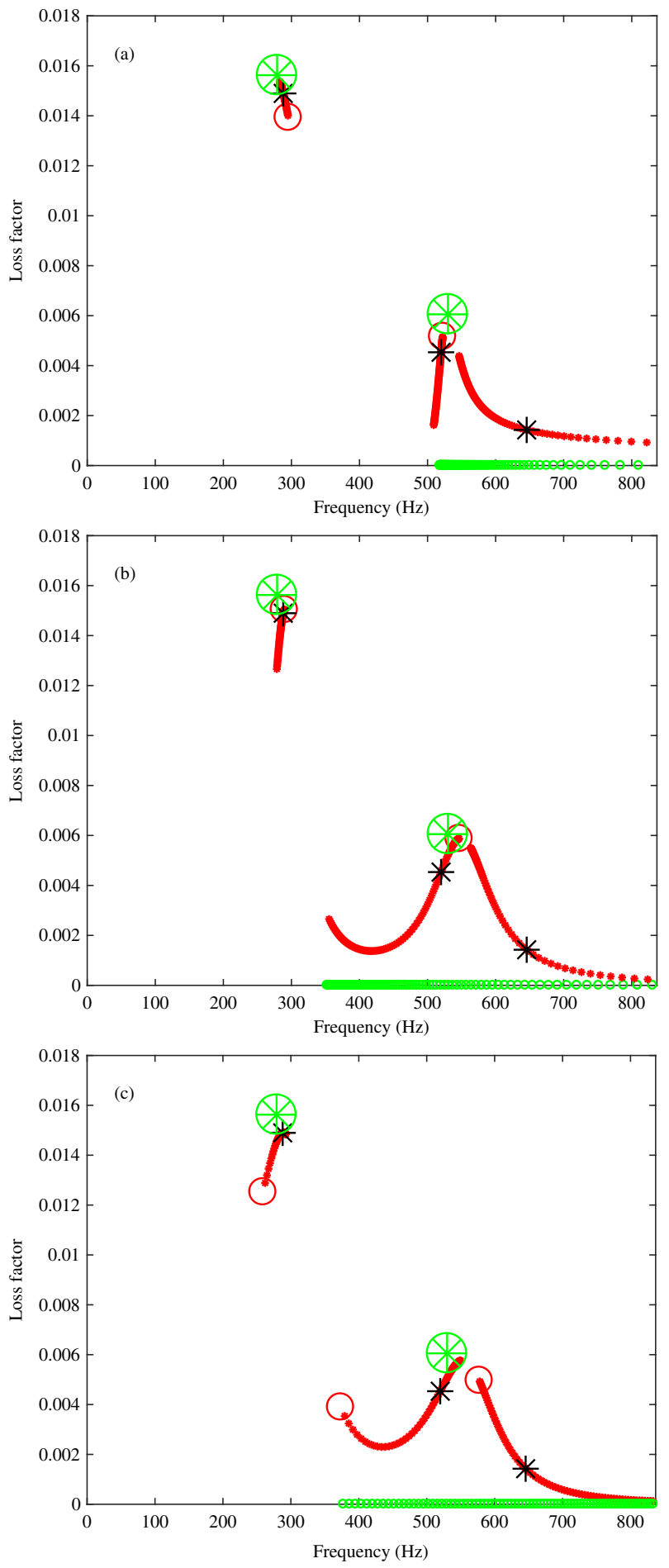

Figure 16. Root-locus plots as explained in the text, for the cases (a) $C$ is varied from zero to $3 \times 10^{-4} \mathrm{~m} / \mathrm{N}$; (b) $m_{b}$ is varied from zero to $6.6 \mathrm{~g}$; (c) $k_{s}$ is varied from zero to $60 \mathrm{kN} / \mathrm{m}$. Circles indicate the zero value of each parameter. Black stars: nominal case; large green symbols: first two fitted poles of the baremembrane admittance; green stars along the horizontal axis: values of $\Omega$ corresponding to the range investigated.

pole would also tend towards that original frequency, but if $\omega_{b}$ were reduced below $\omega_{1}$ it would head, very slowly, towards zero frequency. 
This pattern agrees neatly with behaviour analysed by Langley and Keane [32]. They considered the case of a rigid circular mass at the centre of an ideal membrane, and computed modal behaviour for a range of values of the mass radius. They had no added stiffness, so $\omega_{b}=0$. They showed that as the mass radius tends to zero, one mode tends slowly towards zero frequency, while the frequencies and mode shapes of all the other modes tend towards those of a membrane clamped at the centre: in the limit, the mass acts as a point constraint. Recall from Figure 9 that changing the radius is equivalent to changing the static compliance $C^{\prime}$. The behaviour revealed by Figure 16a also explains a slightly counter-intuitive result: in an extreme case it might appear that adding a concentrated mass to a membrane results in the frequency going up, rather than down as expected.

A somewhat similar pattern is seen in the other two cases in Figure 16. Case (b) shows the effect of varying $m_{b}$. The main difference from case (a) is that the range of $\Omega$ now approaches $\omega_{1}$, so that a somewhat stronger effect is seen on the lowest pole. Case (c) shows the effect of varying $k_{s}$. In this case, the frequencies move up rather than down as $k_{s}$ increases, so that all three initial values when $k_{s}=0$ appear in the plot.

One important thing is clear in all three plots in Figure 16. The loss factors corresponding to the original pair of bare-membrane modes lie higher in the plot than any of the nearby points on the root locus branches. When a pole lies a long way from either original resonance, the corresponding loss factor is significantly lower. This explains a major feature seen in Figure 11: the effect of adding a point mass or stiffness, in the presence of a significant term in $C^{\prime}$, is to reduce damping, and thus produce unexpectedly high peaks in the admittance. The situation with the real banjo bridge is more complicated, but the results seen in Figure 13 suggest that traces of this behaviour carry over to that case. The ad hoc addition of a damper, as in Figures 11c and 11d, gives a possible pragmatic way to ameliorate over-low damping. This issue becomes very significant when the bridge admittance is used for sound synthesis, as was illustrated and discussed in the earlier paper [1].

\section{Conclusions}

The vibration behaviour of an American 5-string banjo has been studied, both experimentally and theoretically. Measurements of the mechanical admittance at the bridge showed a strong contrast with corresponding measurements on a guitar [1]. Furthermore, the addition of the bridge and strings to the banjo was shown to make a very large difference compared to the behaviour of the bare membrane. In this paper, the physics lying behind these measured results has been explored.

A variety of modelling techniques has been applied to the problem. These range from a detailed Finite Element/ Boundary Element model of the banjo and surrounding air, through a simple membrane model based on rectangular geometry and analytical results, to low-order modelling to explore sensitivity to parametric changes. These approaches have given different insights, leading to an overall picture that seems fairly complete.

The stretched membrane of a banjo contrasts with instruments like the guitar which use a structure based on wooden plates. Three distinctive features of the dynamic behaviour of a membrane have been shown to be important for banjo acoustics.

First, the modal density behaves differently: any plate has constant modal density (on average), whereas a membrane has a modal density that grows linearly with frequency. This changes the level and trend of the frequency response, accounting for the fact that the admittance of the bare membrane of a banjo exceeds that of a guitar by upwards of $40 \mathrm{~dB}$ in the low $\mathrm{kHz}$ frequency range. This contrast can be revealed clearly by Skudrzyk's mean value method.

Second, the sound radiation from an air-loaded membrane behaves quite differently from a plate. Any banjo or drum membrane has a wave speed slower than the speed of sound in air, but this speed is independent of frequency and there is no equivalent of the critical frequency of an air-loaded plate. The result is that the radiation efficiency, and radiation damping, of a membrane is highest at low frequency and tends to fall with increasing frequency: the opposite trend from a plate.

Finally, the response of an ideal membrane to a point force exhibits a logarithmic singularity. Naturally, in a real membrane something acts to make the response finite, but there is a legacy of the suppressed singularity in sensitivity of behaviour to perturbations.

When the bridge and strings are added to the bare membrane, the admittance is strongly affected. For frequencies up to about $3 \mathrm{kHz}$, bridge admittance is dominated by a formant. Its centre frequency and bandwidth are determined by the mass of the bridge, and a combination of two sources of stiffness: one associated with the static compliance of the membrane, the other with a combination of effects of string tension, axial stiffness of the strings and in-plane stiffness of the membrane, all sensitive to the break angle of the strings over the bridge. This formant imposes an amplitude envelope on the peaks arising from the membrane modes, which can be analysed successfully using point-coupling theory and measured admittance of the bare membrane, or using the rectangular membrane model. Skudrzyk's mean value method can be used to give a synoptic view of how the formant is affected by the system parameters.

At frequencies higher than about $3 \mathrm{kHz}$, more elaborate modelling is needed. The FE/BE model has been shown to agree reasonably well with the measurements over a wide range of frequency. It reveals that the tested banjo, with a standard three-footed bridge, exhibits a phenomenon closely related to the "bridge hill" which has been much studied in the violin. A combination of the intrinsic dynamics of the bridge and the effect of coupling to the membrane through the feet produces two formant-like features. One occurs in this particular banjo around $5 \mathrm{kHz}$, and is related 
to bending of the bridge, while another occurs around $3 \mathrm{kHz}$ and is associated with bridge deformation illustrated in Figure 14a. It is likely that these banjo bridge hills provide a resource for tonal adjustment, as is known to be the case in the violin.

Combining these two hill features with the lowfrequency formant discussed earlier leads to the following summary of the vibration behaviour of the banjo. The membrane provides many modes, with a modal density that increases with frequency. The amplitudes of these modes in the bridge admittance is shaped by three formants: one at low frequency, plus the two bridge hills. The admittance of the banjo tends to be far higher than that of a guitar, so that the coupling of the body to the strings is higher. The radiation damping tends to decrease with increasing frequency, but the intrinsic material damping of the membrane is far lower than that of a guitar soundboard, or any other wooden structure. The combined result of these factors is that the proportion of the energy that ends up being radiated as sound is far higher than for the guitar, for a given amount put into string vibration by the player.

\section{Acknowledgments}

Many people have contributed to this study: the authors thank Julien Legault, Vincent Cotoni, Robin Langley, Evan Davis, Brian Moore, Martin Woodhouse, Alan Heaver, and the Deering Banjo Company. We also thank three reviewers for comments leading to significant improvements.

\section{Conflict of interest}

The authors declare that they have no conflict of interest.

\section{Data availability statement}

The research data associated with this article are included within the article.

\section{References}

1.J. Woodhouse, D. Politzer, H. Mansour: Acoustics of the banjo: measurements and sound synthesis. Acta Acustica 5, 15 (2021). https://doi.org/10.1051/aacus/2021009.

2. Deering Banjo Company. [Online] Available at: https:// www.deeringbanjos.com/ [Accessed: Dec 29 2020].

3. Martin Woodhouse Guitars. [Online] Available at: http:// www.woodhouse-guitars.co.uk/ [Accessed: Dec 29 2020].

4. Euphonics: The science of musical instruments. [Online] Available at: https://euphonics.org/ [Accessed: Dec 29 2020].

5.E. Durup, E.V. Jansson: The quest of the violin bridgehill. Acta Acustica United with Acustica 91, 2 (2005) 206-213.
6. E. Jansson, B. Niewczyk: On the acoustics of the violin: Bridge hill or body hill? Journal of the Catgut Acoustical Society 3, 7 (1999) 23-27.

7. J. Woodhouse: On the "bridge hill" of the violin. Acta Acustica United with Acustica 91, 1 (2005) 155-165.

8. G. Weinreich, in: Mechanics of musical instruments. Hirschberg A., Kergomard J., Weinreich G., Editors. New York: Springer-Verlag, 1995, pp. 79-114.

9. A. Chaigne, J. Kergomard: Acoustics of musical instruments. Springer Verlag, New York, 2013.

10. J.W.S. Rayleigh: The theory of sound. Macmillan and Co., London, 1877.

11. C.H. Hodges, J. Woodhouse: Theories of noise and vibration transmission in complex structures. Reports on Progress in Physics 49, 2 (1986) 107-170.

12. R.H. Lyon, R.G. DeJong: Theory and application of statistical energy analysis, 2nd ed. Butterworth-Heinemann, Boston, 1995.

13. P.M. Morse, K.U. Ingard: Theoretical acoustics. Princeton University Press, Princeton, NJ, 1986.

14. E. Skudrzyk: The mean-value method of predicting the dynamic-response of complex vibrators. Journal of the Acoustical Society of America 67, 4 (1980) 1105-1135.

15. B. Elie, F. Gautier, B. David: Macro parameters describing the mechanical behavior of classical guitars. Journal of the Acoustical Society of America 132 (2012) 4013-4024.

16. J. Woodhouse, R.S. Langley: Interpreting the input admittance of violins and guitars. Acta Acustica United with Acustica 98, 4 (2012) 611-628.

17. A.P. Dowling, J.E. Ffowcs Williams: Sound and sources of sound. Ellis Horwood, Chichester, 1983.

18. L. Cremer: The physics of the violin. MIT Press, Cambridge, MA, 1984.

19. O. Christensen, B.B. Vistisen: Simple model for lowfrequency guitar function. Journal of the Acoustical Society of America 68 (1980) 756-766.

20. W.M. Leach: Introduction to electroacoustics \& audio amplifier design. 4th ed. Kendall Hunt, Dubuque, Iowa, 2009.

21. D. Politzer, The open back of the open-back banjo. [Online] Available at: http://www.its.caltech.edu/ politzer/TheOpenBackOfTheOpenBack.pdf [Accessed: Dec 29 2020].

22. L. Cremer, M. Heckl, B.A.T. Petersson: Structure-borne sound : structural vibrations and sound radiation at audio frequencies, 3rd ed. Springer, Berlin; New York, 2005.

23. R.R. Christian, R.E. Davis, A. Tubis, C.A. Anderson, R.I Mills, T.D. Rossing: Effects of air-loading on timpani membrane vibrations. Journal of the Acoustical Society of America 76 (1984) 1336-1345.

24. F.G. Leppington, E.G. Broadbent, K.H. Heron: The acoustic radiation efficiency of rectangular panels. Proceedings of the Royal Society of London Series A - Mathematical Physical and Engineering Sciences 382 (1982) 245-271.

25. J. Woodhouse: Plucked guitar transients: Comparison of measurements and synthesis. Acta Acustica United with Acustica 90, 5 (2004) 945-965.

26. F. Fahy, P. Gardonio: Sound and structural vibration: radiation, transmission and response, 2nd ed. Elsevier/ Academic, Amsterdam/Boston, 2007.

27. wave ${ }^{6}$ software: Release 2020.3.4. [Online] Available at: https://www.3ds.com/products-services/simulia/products/ wave6/ [Accessed: Dec 29 2020].

28. R.S. Langley: Numerical evaluation of the acoustic radiation from planar structures with general baffle conditions using wavelets. Journal of the Acoustical Society of America 12 (2007) 766-777.

29. B.C.J. Moore: An introduction to the psychology of hearing, 6th ed. Academic Press, Amsterdam; Boston, 2013. 
30. F.J. Dyson: The radiation theories of Tomonaga, Schwinger and Feynman. Physical Review 75 (1949) 486-502.

31. J. Rae: The science of string instruments. In: T.D. Rossing, Editor. Springer-Verlag, New York, 2010, pp. 59-75.
32. R.S. Langley, A.J. Keane: The effect of attachments on the natural frequencies of a membrane. Journal of Sound and Vibration 188 (1995) 760-766.

Cite this article as: Woodhouse J, Politzer D \& Mansour H. 2021. Acoustics of the banjo: theoretical and numerical modelling. Acta Acustica, 5, 16. 